\title{
Rigorous Results for the Free Energy in the Hopfield Model
}

\author{
S. Albeverio ${ }^{1,3}$, B. Tirozzi $^{2}$ and B. Zegarlinski ${ }^{1}$ \\ 1 Department of Mathematics, University of Bochum; SFB 237 (Essen-Bochum-Düsseldorf), \\ D-4630 Bochum 1, FRG \\ ${ }^{2}$ Department of Mathematics, University of Rome, Rome, Italy \\ ${ }^{3}$ BiBoS Bielefeld; CERFIM Locarno
}

Received July 13, 1991; in revised form February 17, 1992

\begin{abstract}
We prove that the free energy of the Hopfield model with a finite number of patterns can be represented in terms of an asymptotic series expansion in inverse powers of the neurons number. The series is Borel summable for large temperatures. We also establish mathematically some other interesting properties, partly used before in a seminal paper by Amit, Gutfreund and Sompolinsky.
\end{abstract}

\section{Introduction}

One of the first papers in which the critical temperature of the Hopfield model and the properties of the overlaps were intensively discussed is "Spin-glass models of neural networks" by D.J. Amit, H. Gutfreund and H. Sompolinsky [1]. In the first part of that paper the expression of the free energy was deduced in the limit $N \rightarrow \infty$ applying heuristically the saddle point technique to the expression of the partition function, for $N<\infty$. The results of that calculation were very interesting and many of them have been checked also by numerical simulations. Obviously it was not the aim of that paper to prove mathematically the results presented. In the present paper, besides providing mathematical proofs, we also deduce new stronger properties of the free energies. Let us briefly describe our main results, referring to Sects. 2 and 3 for proofs. Consider the Hamiltonian of the Hopfield model [8] with p patterns:

$$
H_{N}(\xi)=\frac{-1}{2 N} \sum_{\mu=1}^{p} \sum_{i \neq j, i, j=1}^{N} \xi_{i}^{\mu} \xi_{j}^{\mu} \sigma_{i} \sigma_{j},
$$

where $\sigma_{i}, i=1, \ldots, N$ are the neuronal activities, $\sigma_{i}= \pm 1$, and

$$
\xi_{i}^{\mu}, \quad i=1, \ldots, N, \quad \xi_{i}^{\mu}= \pm 1
$$

is the codification of the $\mu^{\text {th }}$ pattern which we want to memorize, $\mu=1, \ldots, p$.

All the $\xi_{i}^{\mu}$ are considered to be random variables independent and equally distributed with probability $E\left\{\xi_{i}^{\mu}= \pm 1\right\}=\frac{1}{2}$. The retrieval property of the Hopfield model corresponds to the fact that the "mean value" of the "spins" is 
aligned with some of the $p$ patterns $\xi_{i}^{\mu}, i=1, \ldots, N$. By "mean value" of the "spins," by definition, we mean that the variables $\sigma_{i}, i=1, \ldots, N$ are random variables whose probability distribution $P_{N}$ is described by the Gibbs distribution defined by the Hamiltonian $H_{N}(\xi)$, for finite $N$ and a given inverse temperature $\beta$, and that we take the average of $\sigma_{i}$ with respect to $P_{N}$.

It is interesting to have information also about the property of retrieval averaged with respect to all possible choices of $\xi_{i}^{\mu}, i=1, \ldots, N$ and so one looks for an equation which determines the values of the overlap (order) parameters:

$$
m_{\mu}=\frac{1}{N} E \sum_{i=1}^{N} \xi_{i}^{\mu}\left\langle\sigma_{i}\right\rangle,
$$

where $E$ here means expectation with respect to the probability distribution of the variables $\xi_{i}^{\mu}$ and $\langle\cdot\rangle$ is the expectation with respect to the Gibbs distribution defined above. We say that the system retrieves a pattern when

$$
m_{\mu}=1
$$

Let us define as usual the neural dynamics for the discrete time $t=0,1,2, \ldots$ by

$$
\sigma_{i}(t+1)=\operatorname{sign}\left(\sum_{j=1}^{N} J_{i, j} \sigma_{j}(t)\right)
$$

with given $\sigma_{j}(0)$ and where the couplings ("synaptic efficacities") $J_{i, j}$ - the same as in the Hamiltonian (1.1) - are given by Hebb's rule as follows:

$$
J_{i, j}=\frac{1}{N} \sum_{\mu=1}^{p} \xi_{i}^{\mu} \xi_{j}^{\mu} .
$$

The pattern $\xi_{j}^{\mu}$ are expected to be invariant (stable) with respect to this dynamics only in the thermodynamic limit $N \rightarrow \infty$, hence it is interesting to compute the above quantities (1.2) in the thermodynamic limit. In particular one tries to compute the mean free energy of the system as a function of the temperature and of the $m_{\mu}$. The determination of its local minima should yield then the physical values of the patterns overlap $m_{\mu}$. Let us introduce the partition function of the system:

$$
\mathrm{Z}_{N}(\xi)=\frac{1}{2^{N}} \sum_{\sigma_{i}, i=1, \ldots, N} \exp \left(-\beta H_{N}(\xi)\right)
$$

with a positive constant $\beta$, and then compute the averaged free energy in the thermodynamic limit $N \rightarrow \infty$ :

$$
f=\lim _{N \rightarrow \infty} E F_{N}(\xi)
$$

where

$$
F_{N}(\xi) \equiv \frac{1}{N} \ln Z_{N}(\xi)
$$


Following '[1], one can express the partition function $Z_{N}(\xi)$ as a $p$-dimensional integral using the properties of Gaussian integrals:

$$
\begin{aligned}
Z_{N}(\xi)= & e^{-\beta p / 2} \frac{1}{2^{N}} \sum_{\substack{\sigma_{i} \\
i=1, \ldots, N}}(2 \pi)^{-p / 2} \int d m^{1} \ldots d m^{p} \\
& \times \exp \left\{-\frac{1}{2} \sum_{\mu=1}^{p}\left(m^{\mu}\right)^{2}+\sqrt{\frac{\beta}{N}} \sum_{\mu=1}^{p} m^{\mu} \sum_{i=1}^{N} \sigma_{i} \xi_{i}^{\mu}\right\} \mid
\end{aligned}
$$

In [1] the last sum in the exponential is substituted "by the expectation $E$ with respect to all the $\xi^{\mu}$ in the limit $N \rightarrow \infty$," i.e. in [1] the following quantity $Z_{N}$ is studied

$$
\begin{aligned}
Z_{N}= & e^{-\beta p / 2}(2 \pi)^{-p / 2} \int d m^{1} \ldots d m^{p} \\
& \times \exp \left\{-\frac{1}{2} \sum_{\mu=1}^{p}\left(m^{\mu}\right)^{2}+N E \ln \cosh \left(\sqrt{\frac{\beta}{N}} \sum_{\mu=1}^{p} m^{\mu} \xi^{\mu}\right)\right\} .
\end{aligned}
$$

The legitimation for replacing $Z_{N}(\xi)$ in (1.8) by $Z_{N}$ in (1.9) is not given in [1]. In Sect. 2 we shall provide this legitimation; in fact we prove the $E$-a.e. convergence of the free energy associated with the partition function defined in (1.8) to the free energy associated with $Z_{N}$ (Theorem 2.1). Let us note that part a) of the results in Theorem 2.1 also follows from results obtained by a different (large deviation) method by van Hemmen, see [16]. (See also [17, 9] and references therein for further interesting discussions, and some extensions to other related models; see also $[5,15]$ and $[11,12,18]$.) A further result, proven in Sect. 3 , is that the free energy can be expanded in an asymptotic series in the parameter $1 / N$. The same results hold for the free energy of a system with additional symmetry breaking field (necessary to consider for computation of expectations $\left\langle\sigma_{i}\right\rangle$ used to define the overlap parameters in (1.2)). In Sect. 4 we prove the Borel summability of the expansion for $0<\beta<1$. The notations used in this paper are introduced in Sect. 2 and are motivated by the formulas used in the Introduction.

\section{A Convergence Result for Free Energies}

Let us define, for $\xi=\left(\xi^{i}, i=1, \ldots, N\right), \xi^{i} \in\{-1,+1\}^{p}, m \in R^{p}$ :

$$
S_{N}(m, \xi)=\frac{1}{2} m^{2}-\frac{1}{N} \sum_{i=1}^{N} \ln \cosh \left(\beta^{1 / 2} m \cdot \xi^{i}\right)
$$

with $m^{2}=m \cdot m, \cdot$ being the scalar product in $R^{p}$. $\xi$ stands for $\left(\xi^{1}, \ldots, \xi^{N}\right)$. The space $\{-1,+1\}^{p}$ consists of $2^{p}$ points which we indicate by $\xi_{a}, a=1 \ldots 2^{p}$. Let

$$
q_{a, N}(\xi)=\frac{1}{N} \sum_{i=1}^{N} \delta_{\xi^{2}, \xi_{a}}
$$

with $\delta_{\xi^{i}, \xi_{a}}=1$ for $\xi^{i}=\xi_{a}$ and 0 if $\xi^{i} \neq \xi_{a}$. Thus $q_{a, N}$ is the frequency with which a given $\xi_{a}$ appears in $\left(\xi^{1}, \ldots, \xi^{N}\right)$. We have

$$
S_{N}(m, \xi)=\frac{1}{2} m^{2}-\sum_{a=1}^{2^{p}} q_{a, N}(\xi) \ln \cosh \left(\beta^{1 / 2} m \cdot \xi_{a}\right) .
$$


Let

$$
S(m)=\frac{1}{2} m^{2}-\sum_{a=1}^{2^{p}} \frac{1}{2^{p}} \ln \cosh \left(\beta^{1 / 2} m \cdot \xi_{a}\right) .
$$

Let $\mathbf{E}(\cdot)$ be the expectation with respect to the variable $\xi$. Then

$$
\mathbf{E}\left(\delta_{\xi^{i}, \xi_{a}}\right)=\frac{1}{2^{p}}
$$

the $\delta_{\xi^{i}, \xi_{a}}$ being independent identically distributed. Define

$$
f_{N}(\xi) \equiv \frac{1}{N} \ln \int d m \exp \left(-N S_{N}(m, \xi)\right)=F_{N}(\xi)-\frac{p}{2 N} \ln N
$$

and

$$
\tilde{f}_{N} \equiv \frac{1}{N} \ln \int d m \exp (-N S(m))=\tilde{F}_{N}-\frac{p}{2 N} \ln \mathrm{N}
$$

with $\tilde{F}_{N} \equiv \frac{1}{N} \ln Z_{N}$

Remark. Since

$$
S_{N}(m, \xi) \geqq \frac{1}{2} m^{2}-\beta^{1 / 2} p^{1 / 2}|m|
$$

we have that $f_{N}(\xi)$ is uniformly bounded in $N$ and $\xi$; see Proposition 2.5 below.

The main result of this section is formulated in the following theorem.

Theorem 2.1. For any $0 \leqq \beta<\infty$,

a)

$$
\lim _{N \rightarrow \infty}\left|f_{N}(\xi)-\tilde{f}_{N}\right|=0, \quad \text { E-a.s. }
$$

b) Moreover, there are positive constants $c_{1}, c_{2}, \gamma$ such that

$$
\mathbf{E}\left|f_{N}(\cdot)-\tilde{f}_{N}\right| \leqq \frac{c_{1}}{N^{1 / 2}}+\frac{c_{2}}{N} \exp (-\gamma N)
$$

for any $N \in \mathbb{N}$.

c) If $0 \leqq \beta<1$, then

$$
\lim _{N \rightarrow \infty} \tilde{f}_{N}=0,
$$

and additionally, if $0 \leqq \beta p<1$, then for any $\xi \in\{-1,+1\}^{p}$

$$
\lim _{N \rightarrow \infty} f_{N}(\xi)=0
$$


Proof. The proof of Theorem $2.1 \mathrm{a}$ ) and b) is based on the following lemma:

Lemma 2.2. For any $0<\varepsilon<1$

$$
\left|f_{N}(\xi)-\frac{1}{N} \ln \int d m \chi\left(|m|<R_{\varepsilon}\right) \exp \left(-N S_{N}(m, \xi)\right)\right| \leqq \frac{C}{N} \exp (-\gamma N)
$$

for some constants $0<C, \gamma<\infty$ independent of $N \in \mathbf{N}$ and $\xi \in\{-1,+1\}^{p}$, and where

$$
R_{\varepsilon} \equiv \frac{2 \beta^{1 / 2} p^{1 / 2}}{\varepsilon} .
$$

Here $\chi(|m|<A)$ denotes the characteristic function of the set $\left\{m \in \mathbf{R}^{p},|m|<A\right\}$. The same bound holds with $f_{N}$ replaced by $\tilde{f}_{N}$ and $S_{N}$ replaced by $S$.

The proof of Lemma 2.2 will be given later. Using Lemma 2.2 we see that

$$
\left|f_{N}(\xi)-\tilde{f}_{N}\right| \leqq\left|f_{N, \varepsilon}(\xi)-\tilde{f}_{N, \varepsilon}\right|+\frac{2 C}{N} \exp (-\gamma N)
$$

with

$$
f_{N, \varepsilon}(\xi)=\frac{1}{N} \ln \int d m \chi\left(|m| \leqq R_{\varepsilon}\right) \exp \left(-N S_{N}(m, \xi)\right)
$$

and

$$
\tilde{f}_{N, \varepsilon}=\frac{1}{N} \ln \int d m \chi\left(|m| \leqq R_{\varepsilon}\right) \exp (-N S(m))
$$

Writing

$$
\exp \left(-N S_{N}(m, \xi)\right)=\exp (-N S(m)) \exp \left(-N\left(S_{N}(m, \xi)-S(m)\right)\right.
$$

in the definition of $f_{N, \varepsilon}(\xi)$ and using the fact that for $|m|<R_{\varepsilon}$

$$
\begin{aligned}
\left|S_{N}(m, \xi)-S(m)\right| & =\left|\sum_{a=1}^{2^{p}}\left(q_{a, N}(\xi)-\frac{1}{2^{p}}\right) \ln \cosh \left(\beta^{1 / 2} m \cdot \xi_{a}\right)\right| \\
& \leqq \frac{2 \beta p}{\varepsilon} \sum_{a=1}^{2 p}\left|q_{a, N}(\xi)-\frac{1}{2^{p}}\right|,
\end{aligned}
$$

we obtain:

$$
\tilde{f}_{N, \varepsilon}-\frac{2 \beta p}{\varepsilon} \sum_{a=1}^{2^{p}}\left|q_{a, N}(\xi)-\frac{1}{2^{p}}\right| \leqq f_{N, \varepsilon}(\xi) \leqq \tilde{f}_{N, \varepsilon}+\frac{2 \beta p}{\varepsilon} \sum_{a=1}^{2^{p}}\left|q_{a, N}(\xi)-\frac{1}{2^{p}}\right| .
$$

This implies that

$$
\left|f_{N, \varepsilon}(\cdot)-\tilde{f}_{N, \varepsilon}\right| \leqq \frac{2 \beta p}{\varepsilon} \sum_{a=1}^{2^{p}}\left|q_{a, N}(\cdot)-\frac{1}{2^{p}}\right| .
$$


Using the fact that for any $a=1, \ldots, 2^{p}$ we have

$$
\lim _{N \rightarrow \infty}\left|q_{a, N}(\xi)-\frac{1}{2^{p}}\right|=0, \quad \text { E-a.s. }
$$

together with (2.19) and (2.14) we conclude the first part of Theorem 2.1.

To show part b) of Theorem 2.1 we observe that by taking the expectation with respect to the measure $\mathbf{E}$ of both sides of (2.19) and using the fact (proven in Lemma A.3a of the Appendix) that for any $a=1, \ldots, 2^{p}$,

$$
\mathbf{E}\left|q_{a, N}(\cdot)-\frac{1}{2^{p}}\right| \leqq\left(\frac{1}{2^{p}}-\frac{1}{2^{2 p}}\right)^{1 / 2} \frac{1}{N^{1 / 2}},
$$

we get

$$
\mathbf{E}\left|f_{N, \varepsilon}(\cdot)-\tilde{f}_{N, \varepsilon}\right| \leqq \frac{2 \beta p}{\varepsilon} 2^{p}\left[\frac{1}{2^{p}}-\frac{1}{2^{2 p}}\right]^{1 / 2} \frac{1}{N^{1 / 2}} .
$$

This, together with (2.14) yields:

$$
\mathbf{E}\left|f_{N}(\cdot)-\tilde{f}_{N}\right| \leqq \frac{2 \beta p}{\varepsilon}\left(2^{2 p}-1\right)^{1 / 2} \frac{1}{N^{1 / 2}}+2 \frac{C}{N} \exp (-\gamma N),
$$

which ends the proof of part b) of Theorem 2.1.

Remark. Let us note that also in [9] one may find some different arguments showing part a) of Theorem 2.1.

Now we shall prove c). To prove (2.10) let us observe that

$$
\begin{aligned}
\mathbf{E} \ln \cosh \left(\beta^{1 / 2} m \cdot \xi_{0}\right) & =\int_{0}^{1} d t \int_{0}^{t} d t^{\prime} \beta \mathbf{E}\left[\left(m \cdot \xi_{0}\right)^{2}\left(\cosh \left(t^{\prime} \beta^{1 / 2} m \cdot \xi_{0}\right)\right)^{-2}\right] \\
& \leqq \frac{1}{2} \beta \mathbf{E}\left(m \cdot \xi_{0}\right)^{2}=\frac{1}{2} \beta m^{2}
\end{aligned}
$$

Therefore the action $S(m)$, used to define $\tilde{f}_{N}$ in (2.7), satisfies the following inequalities:

$$
\frac{1}{2}(1-\beta) m^{2} \leqq S(m) \leqq \frac{1}{2} m^{2}
$$

Hence, if $0<\beta<1$, we get

$$
\frac{p}{2 N} \ln (2 \pi / N) \leqq \tilde{f}_{N} \leqq \frac{p}{2 N} \ln (2 \pi / N(1-\beta)) .
$$

This implies (2.10). To get (2.11) we write

$$
\begin{aligned}
S_{N}(m, \xi) & \equiv \frac{1}{2} m^{2}-\sum_{a=1}^{2^{p}} q_{a, N}(\xi) \ln \cosh \left(\beta^{1 / 2} m \cdot \xi_{a}\right) \\
& =\sum_{a=1}^{2^{p}} q_{a, N}(\xi)\left(\frac{1}{2} m^{2}-\ln \cosh \left(\beta^{1 / 2} m \cdot \xi_{a}\right)\right)
\end{aligned}
$$


and observe that

$$
\begin{aligned}
\frac{1}{2} m^{2} & \geqq \frac{1}{2} m^{2}-\ln \cosh \left(\beta^{1 / 2} m \cdot \xi_{a}\right) \\
& =\frac{1}{2} m^{2}-\int_{0}^{1} d s \int_{0}^{s} d s^{\prime} \beta\left(m \cdot \xi_{a}\right)^{2}\left(\cosh s^{\prime} \beta^{1 / 2} m \cdot \xi_{a}\right)^{-2} \geqq \frac{1}{2}(1-\beta p) m^{2}
\end{aligned}
$$

Hence we get

$$
\frac{1}{2}(1-\beta p) m^{2} \leqq S_{N} \leqq \frac{1}{2} m^{2}
$$

whence

$$
\frac{p}{2 N} \ln (2 \pi / N) \leqq f_{N}(\xi) \leqq \frac{p}{2 N} \ln (2 \pi / N(1-\beta p)) .
$$

If $0<\beta p<1$, this shows that for any $\xi \in\{-1,+1\}^{p}$ we have

$$
\lim _{N \rightarrow \infty} f_{N}(\xi)=0
$$

This ends the proof of Theorem 2.1.

Now we give a proof of Lemma 2.2.

Proof of Lemma 2.2. Introducing a partition of unity $1=\chi\left(|m| \leqq R_{\varepsilon}\right)+$ $\chi\left(|m|>R_{\varepsilon}\right)$ into the integral in (2.6) we get

$$
\begin{aligned}
f_{N}(\xi)= & \frac{1}{N} \ln \int d m \chi\left(|m| \leqq R_{\varepsilon}\right) \exp \left(-N S_{N}(m, \xi)\right) \\
& +\frac{1}{N} \ln \left[1+\frac{\int d m \chi\left(|m|>R_{\varepsilon}\right) \exp \left(-N S_{N}(m, \xi)\right)}{\int d m \chi\left(|m| \leqq R_{\varepsilon}\right) \exp \left(-N S_{N}(m, \xi)\right)}\right] .
\end{aligned}
$$

From this we see that in order to prove Lemma 2.2 it suffices to show that

$$
\frac{\int d m \chi\left(|m|>R_{\varepsilon}\right) \exp \left(-N S_{N}(m, \xi)\right)}{\int d m \chi\left(|m| \leqq R_{\varepsilon}\right) \exp \left(-N S_{N}(m, \xi)\right)} \leqq C \exp (-N \gamma)
$$

for some $0 \leqq C, \gamma \leqq \infty$, independent of $N \in \mathbf{N}$ and $\xi$. The inequality (2.33) follows from Lemma 2.3 given below by taking

$$
C \equiv \frac{\exp \left(2 \frac{(1-\varepsilon)}{\varepsilon^{2}} \beta p\right) \int d m \exp \left(-\frac{(1-\varepsilon)}{2} m^{2}\right)}{\int d m \chi\left(|m| \leqq\left(2 \frac{(1-\varepsilon)}{\varepsilon^{2}} \beta p\right)^{1 / 2}\right)}
$$

and

$$
\gamma \equiv \frac{(1-\varepsilon)}{\varepsilon^{2}} \beta p
$$

This ends the proof of Lemma 2.2. 


\section{Lemma 2.3.}

$$
\begin{aligned}
& \int d m \chi\left(|m|>R_{\varepsilon}\right) \exp \left(-N S_{N}(m, \xi)\right) \\
& \quad \leqq \int d m \exp \left(-\frac{(1-\varepsilon)}{2} m^{2}\right) \exp \left(-(N-1)(1-\varepsilon) 2 \beta p / \varepsilon^{2}\right)
\end{aligned}
$$

and

$$
\begin{aligned}
& \int d m \chi\left(|m| \leqq R_{\varepsilon}\right) \exp \left(-N S_{N}(m, \xi)\right) \\
& \quad \geqq \int d m \chi\left[|m| \leqq\left(2 \frac{(1-\varepsilon)}{\varepsilon^{2}} \beta p\right)^{1 / 2}\right] \exp \left(-N \frac{(1-\varepsilon)}{\varepsilon^{2}} \beta p\right) .
\end{aligned}
$$

Proof of Lemma 2.3. To prove the first inequality we remark that for $|m|>R_{\varepsilon}$ we have

$$
\frac{1}{2} \varepsilon m^{2}-\sum_{a=1}^{2^{p}} q_{a, N}(\xi) \ln \cosh \left(\beta^{1 / 2} m \cdot \xi_{a}\right) \geqq \frac{1}{2} \varepsilon m^{2}-\beta^{1 / 2} p^{1 / 2}|m| \geqq 0 .
$$

Therefore

$$
\begin{aligned}
& \int d m \chi\left(|m|>R_{\varepsilon}\right) \exp \left(-N S_{N}(m, \xi)\right) \\
& \quad \leqq \int d m \chi\left(|m|>R_{\varepsilon}\right) \exp \left(-\frac{1-\varepsilon}{2} m^{2}\right) \\
& \quad \leqq \exp \left(-(N-1) \frac{1-\varepsilon}{2} R_{\varepsilon}^{2}\right) \int d m \exp \left(-N \frac{1-\varepsilon}{2} m^{2}\right)
\end{aligned}
$$

which is the first inequality in Lemma 2.3.

To get the second inequality in Lemma 2.3 we observe that for any $0<\alpha<R_{\varepsilon}$ we have

$$
\begin{aligned}
& \int d m \chi\left(|m| \leqq R_{\varepsilon}\right) \exp \left(-N S_{N}(m, \xi)\right) \\
& \geqq \int d m \chi(|m|<\alpha) \exp \left(-N \max _{|m|<\alpha} S_{N}(m, \xi)\right) .
\end{aligned}
$$

However

$$
S_{N}(m, \xi)=\frac{1}{2} m^{2}-\sum_{a=1}^{2^{p}} q_{a, N}(\xi) \ln \cosh \left(\beta^{1 / 2} m \cdot \xi_{a}\right) \leqq \frac{1}{2} m^{2}
$$

So taking

$$
\alpha \equiv\left[\frac{1-\varepsilon}{2} R_{\varepsilon}^{2}\right]^{1 / 2},
$$

we get

$$
\max _{|m|<\alpha} S_{N}(m, \xi) \leqq \frac{1-\varepsilon}{\varepsilon^{2}} \beta_{p} .
$$

This together with (2.40) yields the second inequality in Lemma 2.3. 
Remark. If $\beta \geqq 1$, one can show that there exists an open set $U \subset \mathbf{R}^{p}$ independent of $\xi$ such that for any $m \in U$ we have $S_{N}(m, \xi) \leqq A<0$ for some $0<|A|<\infty$. This allows to improve, in this case, the second estimate in Lemma 2.3.

In the following Propositions (2.4)-(2.6) we shall derive some additional information about the free energies. The first one shows that the mean free energy $\mathbf{E} f_{N}$ is always not less than the free energy $\tilde{f}_{N}$.

Proposition 2.4. For any $N \in \mathbb{N}$

$$
\mathbf{E} \frac{1}{N} \ln \int d m e^{-N S_{N}(m, \xi)} \geqq \frac{1}{N} \ln \int d m e^{-N \mathbf{E} S_{N}(m, \xi)} .
$$

Proof. Let us introduce for $t \in[0,1]$ an interpolating action

$$
S_{N, t} \equiv t S_{N}+(1-t) \mathbf{E} S_{N}
$$

We have

$$
\begin{aligned}
\mathbf{E}\left(f_{N}-\tilde{f}_{N}\right) & =\mathbf{E} \int_{0}^{1} d t \frac{d}{d t} \frac{1}{N} \ln \int d m e^{-N S_{N, t}} \\
& =\left.\mathbf{E} \frac{d}{d t} \frac{1}{N} \ln \int d m e^{-N S_{N, t}}\right|_{t=0}+\int_{0}^{1} d t \int_{0}^{t} d t^{\prime} \mathbf{E} \frac{d^{2}}{d t^{\prime 2}} \frac{1}{N} \ln \int d m e^{-N S_{N, t^{\prime}}} \\
& =-\left.\mathbf{E} \rho_{t=0}\left(\frac{d}{d t} S_{N, t}\right)\right|_{t=0}+N \int_{0}^{1} d t \int_{0}^{t} d t^{\prime} \mathbf{E} \rho_{t^{\prime}}\left(\frac{d}{d t^{\prime}} S_{N, t^{\prime}}, \frac{d}{d t^{\prime}} S_{N, t^{\prime}}\right),
\end{aligned}
$$

where

$$
\rho_{t}() \equiv \frac{\int d m\left(e^{-N S_{N, t}} \cdot\right)}{\int d m e^{-N S_{N, t}}} .
$$

It follows from the definition of $\rho_{t}$ and of $S_{N, t}$ that the first term from the rhs of (2.47) vanishes.

On the other hand, as follows from the Hölder inequality, the second term from the rhs of $(2.47)$ is always nonnegative. This ends the proof of Proposition 2.4 .

Now we show that the free energy $f_{N}(\xi)$ is uniformly bounded in $\xi$. We have

Proposition 2.5. There is a constant $0<C_{0}<\infty$ such that for any $N \in \mathbb{N}$ and $\xi \in\{-1,+1\}^{p}$,

$$
\left|f_{N}(\xi)\right|<C_{0}
$$

Proof. Since

$$
\ln \cosh \left(\beta^{1 / 2} m \cdot \xi_{0}\right) \leqq \beta^{1 / 2} p^{1 / 2}|m|
$$

so

$$
S_{N}(m, \xi) \geqq \frac{1}{2} m^{2}-\beta^{1 / 2} p^{1 / 2}|m|
$$


On the other hand

$$
\begin{aligned}
\frac{1}{N} \ln \int d m \exp \left(-N S_{N}(m, \xi)\right)= & \frac{1}{N} \ln \int d m \exp \left(-N m^{2} / 2\right) \\
& +\frac{1}{N} \ln \frac{\int d m \exp \left(-N S_{N}(m, \xi)\right)}{\int d m \exp \left(-N m^{2} / 2\right)},
\end{aligned}
$$

and, by Gaussian integration,

$$
\frac{1}{N} \ln \int d m \exp \left(-N m^{2} / 2\right)=\frac{1}{N} \ln \left(N^{-p / 2}(2 \pi)^{p / 2}\right) .
$$

Using (2.50) we can estimate the second term from (2.51) as follows:

$$
\begin{aligned}
0 & \leqq \frac{1}{N} \ln \frac{\int d m \exp \left(-N S_{N}(m, \xi)\right)}{\int d m \exp \left(-N m^{2} / 2\right)} \leqq \frac{1}{N} \ln \frac{\int d m \exp \left(-N\left(\frac{1}{2} m^{2}-\beta^{1 / 2} p^{1 / 2}|m|\right)\right)}{\int d m \exp \left(-N m^{2} / 2\right)} \\
& \leqq \frac{1}{N} \ln \left[\frac{\int d m^{\alpha} \exp \left(-N\left(\frac{1}{2}\left(m^{\alpha}\right)^{2}-\beta^{1 / 2} p^{1 / 2}\left|m^{\alpha}\right|\right)\right.}{\int d m \exp \left(-N\left(m^{\alpha}\right)^{2} / 2\right)}\right]^{p} \\
& \leqq \frac{1}{N} \ln [2 \exp (\beta p N / 2)]^{p}=\beta p^{2} / 2+\frac{p \ln 2}{N}
\end{aligned}
$$

This yields Proposition 2.5.

Proposition 2.5 together with some large deviation estimates allows us to conclude that to get the essential information about the free energy for large $N$ it is enough to consider only "good" configurations of $\xi$ 's, a fact which will be useful later on. We have

Proposition 2.6. For $\delta>0$, let

$$
\chi_{\delta} \equiv \chi\left(\left|q_{a, N}-\frac{1}{2^{p}}\right| \leqq \delta \quad \forall a=1, \ldots, 2^{p}\right) .
$$

Then

$$
\left|\mathbf{E} f_{N}(\xi)-\mathbf{E}\left(\chi_{\delta} f_{N}(\xi)\right)\right| \leqq C_{0} \mathbf{E}\left(1-\chi_{\delta}\right)=C_{0} C \exp \left(-\delta N^{1 / 2}\right)
$$

for $C_{0}$ as in Proposition 2.5, and some constant $C>0$.

Proof. We use

$$
\mathbf{E} f_{N}(\xi)=\mathbf{E} \chi_{\delta} f_{N}(\xi)+\mathbf{E}\left(1-\chi_{\delta}\right) f_{N}(\xi),
$$

Proposition 2.5, and the large deviation estimate

$$
\mathbf{E}\left(1-\chi_{\delta}\right) \leqq C \exp \left(-\delta N^{1 / 2}\right),
$$

which can be deduced from the definition of $q_{a, N}(\xi)$; see Lemma A.1 in Appendix. 


\section{Asymptotic Expansion for the Free Energy}

In this section we show that the free energies $\tilde{f}_{N}$ and $E f_{N}(\cdot)$ have asymptotic series representations in powers of $N^{-1}$. Let us mention that a general method exists, see e.g. [3], for investigating the Laplace integrals like those used to define the free energies. In principle one could use this method (together with a result of [6]) to obtain an asymptotic expansion for $\tilde{f}_{N}$. Application of this procedure for explicit computation of corresponding coefficients of the series, in general, can be very complicated.

In our work we provide another way, by which we are able to determine in a simple way the required coefficients. Therefore our method can be useful also for numerical studies, which are important in the domain of neural networks. Additionally, with some small modification, our method applies as well to the investigation of the free energy $E f_{N}(\cdot)$. As the reader may have noticed from Sect. 2, the behavior of free energies is essentially different in the high temperature, $0<\beta<1$, and in the low temperature, $\beta>1$, regions. To get our expansions we need also to apply different considerations in both regions (although both cases rely on a common basic idea). Therefore we present them in two subsections beginning from the high temperature case.

3.1. Asymptotic Expansions: High Temperatures. In this subsection we prove the following result.

Theorem 3.1. Let $0<\beta<1$. Then for any $K \in \mathbb{N}$ we have

a)

$$
\tilde{f}_{N}=-\frac{p}{2 N} \ln (N(1-\beta) / 2 \pi)+\tilde{\Sigma}(K)+\tilde{R}_{K+1},
$$

where

$$
\tilde{\Sigma}(K) \equiv \sum_{n=1}^{K} \tilde{c}_{n} N^{-(n+1)},
$$

with the coefficients $\tilde{c}_{n}$ satisfying the bounds

$$
\left|\tilde{c}_{n}\right| \leqq \tilde{C}^{n} n !
$$

for some constant $0<\tilde{C}<\infty$, and the remainder $\tilde{R}_{K+1}$ satisfying

$$
\left.\left|\tilde{R}_{K+1}\right| \leqq \tilde{C}_{K+1} N^{-(K+2}\right)
$$

for some constant $0<\tilde{C}_{K+1}<\infty$.

b) Similarly we have

$$
E f_{N}(\cdot)=-\frac{p}{2 N} \ln (N(1-\beta) / 2 \pi)+\tilde{\Sigma}(K)+\Sigma(K)+R_{K+1},
$$

where the sum $\Sigma(K)$ is defined by

$$
\Sigma(K) \equiv \sum_{n=1}^{K} c_{n} N^{-(n+1)}
$$


with some coefficients $c_{n}$, and the remainder $R_{K+1}$ satisfying the corresponding bound (3.4) with some positive constant $C_{K+1}$ replacing $\tilde{C}_{K+1}$.

Proof of Theorem 3.1. a) For $0<\beta<1$ it is convenient to split the action $S(m)$ from the definition of $\tilde{f}_{N}$ as follows:

$$
S(m) \equiv \frac{1}{2} m^{2}-E \ln \operatorname{ch} \beta^{1 / 2} m \cdot \xi_{0} \equiv \frac{1-\beta}{2} m^{2}+U\left((1-\beta)^{1 / 2} m\right) .
$$

Then changing the integration variables $m \mapsto((1-\beta) N)^{-1 / 2}$, and denoting by $\rho_{0}$ a Gaussian measure on $\mathbb{R}^{p}$ with mean zero and unit covariance we get

$$
\tilde{f}_{N}=-\frac{p}{2 N} \ln (N(1-\beta) / 2 \pi)+\frac{1}{N} \ln \rho_{0}\left(e^{-N U\left(N^{-1 / 2} m\right)}\right)
$$

(for any measure $\rho$ and $\rho$ integrable function $g$ we use the notation $\rho(g) \equiv \int g d \rho$ ). We will show that the second term from the rhs of (3.7) has an asymptotic expansion in powers of $N^{-1}$. To do that we introduce a parameter $t \in[0,1]$ into the exponential in the expectation with measure $\rho_{0}$. Then using Taylor's theorem we obtain the following expansion of the $K^{\text {th }}$ order of the second term from the rhs of (3.7),

$$
\begin{aligned}
\frac{1}{N} & \ln \rho_{0}\left(e^{-N U\left(N^{-1 / 2} m\right)}\right) \\
& =\sum_{k=1}^{K} \frac{(-1)^{k} N^{k-1}}{k !} \rho_{0}(\underbrace{U\left(N^{-1 / 2} m\right), \ldots, U\left(N^{-1 / 2} m\right)}_{k \text {-times }})+\mathcal{O}_{1}(K+1) \\
& \equiv \tilde{\Sigma}_{0}(K)+\mathcal{O}_{1}(K+1)
\end{aligned}
$$

with the remainder

$$
\begin{aligned}
& \mathcal{O}_{1}(K+1) \\
& \equiv(-1)^{K+1} N^{K} \int_{0}^{1} d t_{1} \int_{0}^{t_{1}} d t_{2} \ldots \int_{0}^{t_{K}} d t_{K+1} \\
& \times \rho_{t_{K+1}}(\underbrace{U\left(N^{-1 / 2} m\right), \ldots, U\left(N^{-1 / 2} m\right)}_{K+1 \text {-times }}) .
\end{aligned}
$$

Here we denote by $\rho_{t}$ the probability measure on $\mathbb{R}^{p}$ given by

$$
\rho_{t}(\cdot) \equiv \frac{\rho_{0}\left(e^{-t N U\left(N^{-1 / 2} m\right)} \cdot\right)}{\rho_{0}\left(e^{-t N U\left(N^{-1 / 2} m\right)}\right)}
$$

We used also the notation

$$
\rho_{t}(\underbrace{U\left(N^{-1 / 2} m\right), \ldots, U\left(N^{-1 / 2} m\right)}_{k \text {-times }}) \equiv(-N)^{-k} \frac{d^{k}}{d t^{k}} \ln \rho_{0}\left(e^{-t N U\left(N^{-1 / 2} m\right)}\right)
$$

for the corresponding semi-invariants (cumulants, truncated correlation functions) of the $k^{\text {th }}$ order. Setting $t=0$ in formula (3.11) we get the semi-invariants, which appeared in the sum on the rhs of (3.8), defined with the Gaussian measure $\rho_{0}$.

Let us find an estimate for the remainder $\mathcal{O}_{1}(K+1)$. 
Lemma 3.2. There is a constant $0<D_{K+1}<\infty$ such that

$$
\left|\mathcal{O}_{1}(K+1)\right| \leqq D_{K+1} N^{-(K+2)} .
$$

Proof of Lemma 3.2. Using the definition (3.11) one may easily check that

$$
\left|\rho_{t}\left(U\left(N^{-1 / 2} m\right), \ldots, U\left(N^{-1 / 2} m\right)\right)\right| \leqq 2^{k} k ! \rho_{t}\left(\left|U\left(N^{-1 / 2} m\right)\right|^{k}\right) .
$$

Since by (3.6) and Taylor's theorem we have

$$
\begin{aligned}
U\left(N^{-1 / 2} m\right) & =\frac{1}{2} \frac{\beta}{1-\beta}\left(N^{-1 / 2} m\right)^{2}-E \operatorname{lnch}\left(\left(\frac{\beta}{1-\beta}\right)^{1 / 2}\left(N^{-1 / 2} m\right)\right) \\
& =N^{-2}(\beta /(1-\beta))^{2} \int_{0}^{1} d t_{1} \ldots \int_{0}^{t_{3}} d t_{4} E\left(\left(m \cdot \xi_{0}\right)^{4} u_{4}\left(N^{-1 / 2} m\right)\right)
\end{aligned}
$$

with

$$
\left|u_{4}\left(N^{-1 / 2} m\right)\right| \equiv\left|\frac{d^{4}}{d z^{4}} \ln \operatorname{ch} z\left(z=(\beta / N(1-\beta))^{1 / 2} m \cdot \xi_{0}\right)\right|<C_{1}
$$

for some constant $0<C_{1}<\infty$, so the expectation from the rhs of (3.13) can be estimated as follows:

$$
\rho_{t}\left(\left|U\left(N^{-1 / 2} m\right)\right|^{k}\right) \leqq\left(\frac{C_{1} \beta^{2}}{4 !(1-\beta)^{2} N^{2}}\right)^{k} \rho_{t}\left(\left|E\left(m \cdot \xi_{0}\right)^{4}\right|^{k}\right) .
$$

Using Brascamp-Lieb inequalities [2] for the measure $\rho_{t}$, (defined in (3.10) by perturbation of the Gaussian measure with a log-concave function), together with the Hölder inequality to estimate the expectation from the rhs of (3.16), we obtain the following bound:

$$
\begin{aligned}
\rho_{t}\left(\left|U\left(N^{-1 / 2} m\right)\right|^{k}\right) & \leqq\left(\frac{C_{1} \beta^{2} p^{2}}{4 !(1-\beta)^{2} N^{2}}\right)^{k} \rho_{0}\left(|m|^{4 k}\right) \\
& \leqq\left(\frac{C_{1} \beta^{2} p^{2}}{4 !(1-\beta)^{2}}\right)^{k}\left(\frac{(4 k) !}{2^{4 k}(2 k) !} p^{4 k}\right) N^{-2 k} \\
& \equiv 2^{-k} D_{k} \cdot N^{-2 k}
\end{aligned}
$$

This, together with (3.13), shows that the remainder term $\mathcal{O}_{1}(K+1)$ given by (3.9) satisfies the bound

$$
\left|\mathcal{O}_{1}(K+1)\right| \leqq D_{K+1} N^{-(K+2)}
$$

with the constant $0<D_{K+1}<\infty$ defined in (3.17) for $k=K+1$. This ends the proof of Lemma 3.2.

The above considerations (3.13)-(3.17) show also that each particular term in the sum on the rhs of (3.8) is of order $N^{-(k+1)}$ respectively. (Note however that (3.17) would imply too fast-growth of the coefficients in $k$.) 
Now, to get the expansion described in point a) we have to analyse in more detail the sum $\tilde{\Sigma}_{0}(K)$ from the rhs of (3.8). First we use the representation

$$
U\left(N^{-1 / 2} m\right)=\sum_{l=2}^{K} a_{2 l} N^{-l} E\left(m \cdot \xi_{0}\right)^{2 l}+r_{K+1}(m)
$$

coming from the Taylor expansion to the $K^{\text {th }}$ order. Let us note that, as follows from the properties of the function $\ln \operatorname{ch} x$, the coefficients $a_{2 l}$ from (3.18) satisfy the bound

$$
a^{2 l} \leqq\left|a_{2 l}\right| \leqq A^{2 l}
$$

for some constants $0 \leqq a<A<\infty$. Inserting the expansion (3.16) into the semiinvariants, we obtain the following representation of the sum $\tilde{\Sigma}_{0}(K)$ from the rhs of (3.8):

$$
\begin{aligned}
\tilde{\Sigma}_{0}(K)= & \sum_{k=1}^{K} \frac{(-1)^{k}}{k !} N^{k-1} \sum_{l_{1}, \ldots, l_{k}=2}^{K}\left(\prod_{i=1}^{k} a_{2 l_{i}}\right) N^{-\left(l_{1}+\cdots+l_{n}\right)} \\
& \cdot \rho_{0}\left(E\left(m \cdot \xi_{a}\right)^{2 l_{1}}, \ldots, E\left(m \cdot \xi_{a}\right)^{2 l_{k}}\right)+\mathcal{O}(K+1),
\end{aligned}
$$

where by $\mathcal{O}(K+1)$ we denoted a sum of terms with the function $r_{K+1}(m)$ appearing in at least one of the truncated correlation functions. By similar arguments as those used to estimate $\mathcal{O}_{1}(K+1)$ from (3.9) (given in the proof of Lemma 3.2), we easily get that

$$
|\mathcal{O}(K+1)| \leqq D_{K+1}^{\prime} N^{-(K+2)}
$$

for some constant $0<D_{K+1}^{\prime}<\infty$.

Now by rearranging the terms in the sum on the rhs of (3.20) we obtain

$$
\tilde{\Sigma}_{0}(K)=\sum_{n=1}^{K} c_{n} N^{-(n+1)}+\mathcal{O}_{2}(K+1)
$$

with

$$
c_{n} \equiv \sum_{\substack { k \leqq n \\
\begin{subarray}{c}{k,\left\{l_{1}, \ldots, l_{k} \geqq 2\right\} \\
l_{1}+\cdots,+l_{k}-k=n{ k \leqq n \\
\begin{subarray} { c } { k , \{ l _ { 1 } , \ldots , l _ { k } \geqq 2 \} \\
l _ { 1 } + \cdots , + l _ { k } - k = n } }\end{subarray}} \frac{(-1)^{k}}{k !}\left(\prod_{i=1}^{k} a_{2 l_{i}}\right) \rho_{0}(\underbrace{E\left(m \cdot \xi_{a}\right)^{2 l_{1}}, \ldots, E\left(m \cdot \xi_{a}\right)^{2 l_{k}}}_{k \text {-times }}),
$$

and with $\mathcal{O}_{2}(K+1)$ denoting the sum of $\mathcal{O}(K+1)$ and all other terms from the sum on the rhs of (3.20) which are of order $N^{-(K+1)}$ or smaller. Clearly $\mathcal{O}_{2}(K+1)$ satisfies a bound of the form (3.21) for some other constant $0<D_{K+1}^{\prime \prime}<\infty$. Denoting the first sum from the rhs of (3.22) by $\tilde{\Sigma}(K)$ and using (3.22) and (3.8) we get the representation

$$
\frac{1}{N} \ln \rho_{0}\left(e^{-N U\left(N^{-1 / 2} m\right)}\right)=\tilde{\Sigma}(K)+\tilde{R}_{K+1}
$$

with

$$
\tilde{R}_{K+1} \equiv \mathcal{O}_{1}(K+1)+\mathcal{O}_{2}(K+1) \text {. }
$$

Since $\mathcal{O}_{1}(K+1)$ and $\mathcal{O}_{2}(K+1)$ satisfy bounds as in (3.17) with corresponding constants $D_{K+1}$ and $D_{K+1}^{\prime \prime}$, so $\tilde{R}_{K+1}$ satisfies the bound (3.4) with the constant

$$
\tilde{C}_{K+1} \equiv D_{K+1}+D_{K+1}^{\prime \prime} \text {. }
$$


Now to finish the proof of part a) we have only to prove the bound (3.3) for the coefficients $c_{n}$ given by (3.23). In the proof of this bound, the central role is played by the following lemma, which is a special case of corresponding results proven in $[4]$ (see also $[7,10]$ ) and providing us with estimates of (general) semi-invariants of a Gaussian measure.

Lemma 3.3. There is a constant $0<c<\infty$ such that for any $k \in \mathbb{N}$,

$$
\left|\rho_{0}\left(E\left(m \cdot \xi_{a}\right)^{2 l_{1}}, \ldots, E\left(m \cdot \xi_{a}\right)^{2 l_{k}}\right)\right| \leqq c^{k} k ! \prod_{i=1}^{k}\left(\rho_{0}\left(E\left(m \cdot \xi_{a}\right)^{2 i_{i}}\right)^{2}\right)^{1 / 2}
$$

Using this lemma together with Hölder inequality and simple inequalities for factorials we get the following estimate:

\section{Lemma 3.4.}

$$
\left|\rho_{0}\left(E\left(m \cdot \xi_{a}\right)^{2 l_{1}}, \ldots, E\left(m \cdot \xi_{a}\right)^{2 l_{k}}\right)\right| \leqq c^{k}\left(2 p^{2}\right)^{\left(l_{1}+\cdots+l_{k}\right)} k ! \prod_{i=1}^{k}\left(l_{i} !\right) .
$$

From Lemma 3.4, the estimate (3.19) and the definition of $c_{n}$ in (3.23) we get

$$
\left|c_{n}\right| \leqq \sum_{\substack{k \leqq n,\left\{l_{1}, \ldots, l_{k} \geqq 2\right\} \\ l_{1}+\cdots+l_{k}-k=n}} c^{k}\left(2 A^{2} p^{2}\right)^{\left(l_{1}+\cdots+l_{k}\right)}\left(\prod_{i=1}^{k} l_{i} !\right) .
$$

Changing the summation variables $l_{i} \mapsto l_{i}+1$, one may rewrite (3.28) in the form

$$
\left|c_{n}\right| \leqq \sum_{k \leqq n} \sum_{\substack{l_{1}, \ldots, l_{k} \in \mathbb{N} \\ l_{1}+\ldots+l_{k}=n}} c^{k}\left(2 A^{2} p^{2}\right)^{n+k} \prod_{i=1}^{k}\left(l_{i}+1\right) !
$$

Since for $l_{1}, \ldots, l_{k} \in \mathbb{N}, l_{1}+\cdots+l_{k}=n$ we have

$$
\prod_{i=1}^{k}\left(l_{i}+1\right) !=\prod_{i=1}^{k} l_{i} ! \prod_{i=1}^{k}\left(l_{i}+1\right) \leqq n ! e^{n}
$$

so (3.29) implies the bound

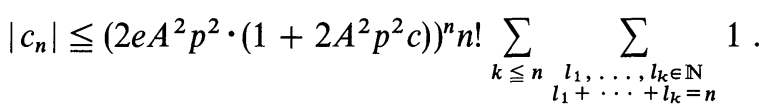

The sum on the rhs of (3.31) is just the number of all possible representations of a positive integer $n$ as a sum of elements of $\mathbb{N}$, which, as is known, equals to $2^{n-1}$. Combining this information with (3.31) we get the inequality (3.3)

$$
\left|c_{n}\right| \leqq \tilde{C}^{n} \cdot n !
$$

with a constant $\tilde{C}$ satisfying the bound

$$
\tilde{C} \leqq 4 e A^{2} p^{2}\left(1+2 A^{2} p^{2}\right) .
$$

This ends the proof of Theorem 3.1a). 
Proof of Theorem 3.1 b). We consider now the free energy

$$
E f_{N} \equiv \frac{1}{N} E \ln \int d m e^{-N S_{N}(m, \xi)}
$$

defined with the action

$$
S_{N}(m, \xi) \equiv \frac{1}{2} m^{2}-\sum_{a=1}^{2^{p}} q_{a, N}(\xi) \ln \operatorname{ch} \beta^{1 / 2} m \cdot \xi_{a} .
$$

Let us note that when $0<\beta<1$, it is not true in general that the point $m=0$ is a maximum point of the action for all configurations $\xi$. (As one may see from the proof of Theorem $2.1 \mathrm{c}$ ), this is the case only when $0<\beta p<1$.) Nevertheless we will make the expansion similarly as before, but additionally taking into account that the probability of "wrong" configurations is small. We begin by splitting the action $S_{N}(m, \xi)$ as follows:

$$
S_{N}(m, \xi) \equiv \frac{1-\beta}{2} m^{2}+U_{\xi, N}\left((1-\beta)^{1 / 2} m\right)
$$

Then changing the integration variables $m \mapsto N^{1 / 2}(1-\beta)^{1 / 2} m$ we get

$$
E f_{N}=-\frac{p}{2 N} \ln (N(1-\beta) / 2 \pi)+\frac{1}{N} E \ln \rho_{0}\left(e^{-N U_{\xi, N}\left(N^{-1 / 2} m\right)}\right) .
$$

Similarly as before we expand the second term to the $K^{\text {th }}$ order. We get

$$
\begin{aligned}
\frac{1}{N} E & \ln \rho_{0}\left(e^{-N U_{\xi, N}\left(N^{-1 / 2} m\right)}\right) \\
= & \frac{1}{N} \sum_{k=1}^{K} \frac{(-1)^{k}}{k !} E \rho_{0}(\underbrace{N U_{\xi, N}\left(N^{-1 / 2} m\right), \ldots, N U_{\xi, N}\left(N^{-1 / 2 m}\right)}_{k \text {-times }}) \\
& +\frac{(-1)^{K+1}}{N} \int_{0}^{1} d t_{1} \ldots \int_{0}^{t_{K}} d t_{K+1} E \rho_{t_{k+1}}(\underbrace{U_{\xi, N}\left(N^{-1 / 2} m\right), \ldots, N U_{\xi, N}\left(N^{-1 / 2} m\right)}_{k+1-\text { times }}) .
\end{aligned}
$$

The probability measure $\rho_{t_{K+1}}$ in (3.38) is defined similarly as in (3.10), but now with the action $U_{\xi, N}$.

Let us denote the first sum from the rhs of (3.38) by $\Sigma_{0}(K)$ and the remainder term by $\mathcal{O}_{1}(K+1)$. We begin by proving the following bound

Lemma 3.5. For any $k \in \mathbb{N}$ there is a constant $C_{1} \equiv C_{1}(k)$ such that

$$
\left|\mathcal{O}_{1}(k)\right| \leqq C_{1} N^{-(1 / 2) k} \text {. }
$$

Proof of Lemma 3.5. Using the definition of truncated correlation functions we get

$$
\begin{gathered}
E \rho_{t}(\underbrace{N U_{\xi, N}\left(N^{-1 / 2} m\right), \ldots, N U_{\xi, N}\left(N^{-1 / 2 m}\right)}_{k \text {-times }}) \mid \\
\leqq k ! E\left(\rho_{t}\left|N U_{\xi, N}\left(N^{-1 / 2} m\right)\right|^{k}\right) .
\end{gathered}
$$


For further purposes it is convenient to introduce the splitting

$$
N U_{\xi, N}\left(N^{-1 / 2} m\right)=\frac{-\beta}{1-\beta} \frac{1}{2} \sum_{a=1}^{2^{p}} \bar{q}_{a, N}\left(m \cdot \xi_{a}\right)^{2}+N V_{\xi, N}\left(N^{-1 / 2} m\right)
$$

with

$$
\bar{q}_{a, N} \equiv q_{a, N}(\xi)-\frac{1}{2^{p}}
$$

and

$$
\begin{aligned}
N V_{\xi, N}\left(N^{-1 / 2} m\right) \equiv & \sum_{a=1}^{2^{p}} q_{a, N}(\xi)\left(\frac{\beta}{1-\beta} \frac{1}{2}\left(m \cdot \xi_{a}\right)^{2}\right. \\
& \left.-N \ln \operatorname{ch}\left(\left(\frac{\beta}{1-\beta}\right)^{1 / 2} N^{-1 / 2}\left(m \cdot \xi_{a}\right)\right)\right) .
\end{aligned}
$$

Now the expectation from the rhs of (3.40) can be estimated as follows:

$$
\begin{aligned}
E \rho_{t}\left(\left|N U_{\xi, N}\left(N^{-1 / 2} m\right)\right|^{k}\right) \leqq & 2^{k-1}\left\{E \rho_{t}\left(\left|\frac{\beta}{1-\beta} \frac{1}{2} \sum_{a=1}^{2^{p}} \bar{q}_{a, N}\left(m \cdot \xi_{a}\right)^{2}\right|^{k}\right)\right. \\
& \left.+E \rho_{t}\left(\left|N V_{\xi, N}\left(N^{-1 / 2} m\right)\right|^{k}\right)\right\} .
\end{aligned}
$$

Let

$$
\chi_{\delta, N} \equiv \chi_{\delta, N}(\xi) \equiv \chi\left\{\left|\bar{q}_{a, N}\right| \leqq \delta: a=1, \ldots, 2^{p}\right\}
$$

with

$$
\delta \equiv N^{-1 / 4} .
$$

Let us first bound the part of the rhs of (3.42) corresponding to the integration over $\xi$ for which $\left|\bar{q}_{a, N}\right|>\delta$. To get a bound corresponding to the second term in the curly bracket in (3.42) we use the Taylor expansion of the function $V_{\xi, N}$ to the fourth order (similarly as in (3.13)-(3.15)). In this way we obtain

$$
\begin{gathered}
E\left(1-\chi_{\delta, N}\right) \rho_{t}\left(\left|N V_{\xi, N}\left(N^{-1 / 2} m\right)\right|^{k}\right) \\
\leqq E\left(1-\chi_{\delta, N}\right)\left(\frac{\beta^{2} p^{2}}{4 !(1-\beta)^{2}} N^{-2}\right)^{k} \sup _{t, \xi} \rho_{t}\left(|m|^{4 k}\right) .
\end{gathered}
$$

To estimate $\rho_{t}\left(|m|^{4 k}\right)$ we insert into the integral the partition of unity similar to the one used in Sect. 2. Then we get

$$
\begin{aligned}
\rho_{t}\left(|m|^{4 k}\right) & =\rho_{t}\left(\chi\left(|m|<N^{1 / 2} R_{\varepsilon}\right)|m|^{4 k}\right)+\rho_{t}\left(\chi\left(|m|>N^{1 / 2} R_{\varepsilon}\right)|m|^{4 k}\right) \\
& \leqq N^{2 k} R_{\varepsilon}^{4 k}+\rho_{t}\left(\chi\left(\mid m>N^{1 / 2} R_{\varepsilon}\right)|m|^{4 k}\right) .
\end{aligned}
$$

Using similar considerations as in the proof of Lemma 2.3. one can show that

$$
\sup _{t, \xi} \rho_{t}\left(\chi\left(|m|>N^{1 / 2} R_{\varepsilon}\right)|m|^{4 k}\right) \leqq C_{2}^{k}(2 k) ! N^{2 k}
$$


for some constant $0<C_{2}<\infty$ independent of $N$ and $k \in \mathbb{N}$. This together with (3.43), (3.44) and Lemma A.2 proven in the Appendix gives us

$$
\begin{aligned}
\sup _{t \in[0,1]} E\left(1-\chi_{\delta, N}\right) \rho_{t}\left(\left|N V_{\xi, N}\left(N^{-1 / 2} m\right)\right|^{k}\right) & \leqq C_{3}^{k}(2 k) ! N^{2 k} E\left(1-\chi_{\delta, N}\right) \\
& \leqq C_{4}^{k}(2 k) ! N^{2 k} e^{-N^{1 / 4}}
\end{aligned}
$$

with some constants $0<C_{3}, C_{4}<\infty$ independent of $N$ and $k$.

The corresponding estimate for the part of the first expectation in curly bracket of the rhs of (3.42) is obtained by using a simple algebraic inequality, translation invariance of $E$ and similar arguments as before. We get

$$
\begin{aligned}
E\left(1-\chi_{\delta, N}\right) \rho_{t}\left(\left|\frac{\beta}{1-\beta} \frac{1}{2} \sum_{a=1}^{2^{p}} \bar{q}_{a, N}\left(m \cdot \xi_{a}\right)^{2}\right|^{k}\right) & \leqq\left(\frac{\beta}{1-\beta} 2^{p-1}\right)^{k} E\left(1-\chi_{\delta, N}\right) \rho_{t}\left(\left|m \cdot \xi_{0}\right|^{2 k}\right) \\
& \leqq C_{5}^{k} k ! N^{k} e^{-N^{1 / 4}}
\end{aligned}
$$

with some constant $C_{5}$ independent of $N$ and $k$. The inequalities (3.40)-(3.47) give the following bound:

$$
\left|E\left(1-\chi_{\delta, N}\right) \rho_{t}(\underbrace{N U_{\xi, N}\left(N^{-1 / 2} m\right), \ldots, N U_{\xi, N}\left(N^{-1 / 2} m\right)}_{k \text {-times }})\right| \leqq C_{6}^{k}(5 k) ! e^{-(1 / 2) N^{1 / 4}}
$$

with some constant $C_{6}$ independent of $N$ and $k$. Let us now bound the part of the lhs of (3.40) corresponding to the integration over $\xi$ 's for which $\left|\bar{q}_{a, N}\right| \leqq \delta$. For this we use (3.40)-(3.42) together with the fact that if we have $\left|\bar{q}_{a, N}\right| \leqq \delta$ with $\delta \equiv N^{-1 / 4}$, then as shown in Lemma 3.8 (at the end of the Sect. 3) the action $S_{N}(m, \xi)$, for $\beta<1$ has the unique minimum at zero and differs from a Gaussian action $\frac{1}{2} \alpha m^{2}$, defined with some positive constant $\alpha$. This last implies (by simple change of integration variables and use of Brascamp-Lieb inequalities) the following bound:

$$
\rho_{t}\left(|m|^{2 k}\right) \leqq C_{7}^{k} k !
$$

with some constant $C_{7}$ independent of $k$. From that we obtain

$$
\begin{aligned}
& E \chi_{\delta, N} \rho_{t}\left(\left|N V_{\xi, N}\left(N^{-1 / 2} m\right)\right|^{k}\right) \\
& \quad \leqq\left(\frac{\beta^{2} p^{2}}{4 !(1-\beta)^{2}} N^{-2}\right)^{k} \sup \left\{\rho_{t}\left(|m|^{4 k}\right): t \in[0,1], \xi \in \operatorname{supp} \chi_{\delta, N}\right\} \\
& \quad \leqq C_{8}^{k}(2 k) ! N^{-2 k}
\end{aligned}
$$

with some constant $C_{8}$ and

$$
\begin{aligned}
& E \chi_{\delta, N} \rho_{t}\left(\left|\frac{\beta}{1-\beta} \frac{1}{2} \sum_{a=1}^{2^{p}} \bar{q}_{a, N}\left(m \cdot \xi_{a}\right)^{2}\right|^{k}\right) \\
& \quad \leqq\left(\frac{\beta}{1-\beta} 2^{p-1}\right)^{k}\left(E\left|\bar{q}_{0, N}\right|^{2 k}\right)^{1 / 2}\left(E \chi_{\delta, N} \rho_{t}\left(\left|m \cdot \xi_{0}\right|^{4 k}\right)\right)^{1 / 2} \\
& \quad \leqq C_{9}^{k} k ! N^{-k / 2}
\end{aligned}
$$

with some constant $C_{9}$ and where in the last step we have used Lemma A3, c) proven in the Appendix. The inequalities (3.50) and (3.51) together with (3.40)(3.42) complete the proof of Lemma 3.5. 
To estimate the rest term $\mathcal{O}_{1}(K+1)$ using the above lemma, we expand it further to the order $2(K+1)$ and apply to the rest term of this expansions inequality (3.39) with $k=2(K+1)$. As follows from the considerations from the proof of Lemma 3.5 applied to the case when $t=0$ the expansion of the rest term $\mathcal{O}_{1}(K+1)$ contains terms which are of order higher than $K+1$ in $N^{-1}$ as well as the terms which are of order less than $K+1$ in $N^{-1}$. Therefore it is clear that to get a final expansion we have to redefine the sum $\Sigma_{0}$ and the rest term in such a way that the final rest term contains only the terms of order higher than $K+1$ with respect to $N^{-1}$. The terms we have to discuss are of the same structure as those from the sum $\Sigma_{0}$. Let us note that as compared to the first case of Theorem 3.1, each term of $\Sigma_{0}$ contains a complicated expectation with respect to $\xi$ 's and therefore we need now to carry out a more complicated analysis involving an additional expansion of each term. We turn now to the more detailed discussion of the sum $\Sigma_{0}$, from which it will also be clear how to deal with the terms obtained by expanding $\mathcal{O}_{1}(K+1)$. For that we use the following representation of the function $N U_{\xi, N}\left(N^{-1 / 2} m\right)$,

$$
\begin{aligned}
& N U_{\xi, N}\left(N^{-1 / 2} m\right)=-\frac{\beta}{1-\beta} \frac{1}{2} \sum_{a=1}^{2 p} \bar{q}_{a, N}\left(m \cdot \xi_{a}\right)^{2} \\
+ & \sum_{a=1}^{2 p} \bar{q}_{a, N}\left(\frac{\beta}{1-\beta} \frac{1}{2}\left(m \cdot \xi_{a}\right)^{2}-N \ln \mathrm{ch}\left[\left(\frac{\beta}{1-\beta}\right)^{1 / 2} N^{-1 / 2} m \cdot \xi_{a}\right]\right) \\
+ & N U\left(N^{-1 / 2} m\right) \equiv W_{\xi, N}(m)+N U\left(N^{-1 / 2} m\right) .
\end{aligned}
$$

Inserting (3.52) into the expression of each term of the sum $\Sigma_{0}(K)$ and using the definition of $\tilde{\Sigma}_{0}(K)$ in (3.8) we obtain

$$
\Sigma_{0}(K)=\tilde{\Sigma}_{0}(K)+\Sigma_{1}(K)
$$

with $\Sigma_{1}(K)$ given by the following expression:

$$
\begin{aligned}
& \sum_{k=1}^{K} \frac{(-1)^{k}}{k !} \sum_{l=1}^{k}\left(\begin{array}{l}
k \\
l
\end{array}\right) N^{k-l-1} \\
& \quad \times E \rho_{0}(\underbrace{W_{\xi, N}(m), \ldots, W_{\xi, N}(m)}_{l}, \underbrace{U\left(N^{-1 / 2} m\right), \ldots, U\left(N^{-1 / 2} m\right)}_{k-l}) .
\end{aligned}
$$

The sum $\tilde{\Sigma}_{0}(K)$ has been investigated before in the proof of point a) of the theorem. To study the second sum from the rhs of (3.54) we use the expansion (3.18) of $W_{\xi, N}$ obtained by application of Taylor's theorem:

$$
W_{\xi, N}(m)=\sum_{a=1}^{2^{p}} \bar{q}_{a, N}(\xi)\left\{\sum_{l=1}^{K-1} a_{2 l} N^{-l+1}\left(m \cdot \xi_{a}\right)^{2 l}+N r_{K, a}\left(N^{-1 / 2} m\right)\right\}
$$

with the coefficients $a_{2 l}$ as in (3.18) (for $l>1$ ) and so satisfying the bound (3.19), and the remainder functions $r_{K, a}, a=1, \ldots, 2^{p}$.

Hence we obtain the representation

$$
\Sigma_{1}(K)=\Sigma_{2}(K)+\mathcal{O}_{2}(K+1)
$$


with the remainder $\mathcal{O}_{2}((K+1))$ and

$$
\begin{aligned}
& \Sigma_{2}(K)=\sum_{k=1}^{K} \frac{(-1)^{k}}{k !} \sum_{j=1}^{k}\left(\begin{array}{l}
k \\
j
\end{array}\right) N^{k-j-1} \\
& \text { - } \sum_{b_{1}, \ldots, b_{j}=1}^{2^{p}} \sum_{l_{1}, \ldots, l_{j}=1}^{K-1} \sum_{l_{j+1}, \ldots, l_{k}=2}^{K} \prod_{i=1}^{k} a_{a_{2 l_{i}}} E\left(\bar{q}_{b_{1}, N} \ldots \bar{q}_{b_{j}, \mathrm{~N}}\right) \\
& \cdot \rho_{0}(\underbrace{\left(m \cdot \xi_{b_{1}}\right)^{2 l_{1}}, \ldots,\left(m \cdot \xi_{b_{j}}\right)^{2 l_{j}}}_{l} \\
& \times \underbrace{E\left(m \cdot \xi_{0}\right)^{2 l_{j+1}}, \ldots, E\left(m \cdot \xi_{0}\right)^{2 l_{k}}}_{k-l}) N^{-\left(\sum_{i=1}^{j}\left(l_{i}-1\right)+\sum_{i=j+1}^{k} l_{i}\right)} .
\end{aligned}
$$

Using the definition of $\mathcal{O}_{2}(\mathrm{~K})$, bounds for Gaussian integrals and estimates of the moments $E \bar{q}_{a, N}^{2 k}$ it is not very difficult to see (by arguments used before) that

$$
\left|\mathcal{O}_{2}(K+1)\right| \leqq D_{1} N^{-(K+2)} \text {. }
$$

Now we have to discuss the sum $\Sigma_{2}(K)$. Let us first note that to get the final expansion it is still not enough to simply rearrange the sum (as was the case in the proof of a)). This is because we have hidden small factors (proportional to some powers of $\left.N^{-1}\right)$ in the expectation $E\left(\bar{q}_{b_{1}, N} \ldots \bar{q}_{b_{j}, N}\right)$. Let us note that (from the analysis given in the Appendix) we have that each term

$$
E\left(\bar{q}_{b_{1}, N} \ldots \bar{q}_{b_{j}, N}\right)
$$

is a polynomial of order at most $N^{-j / 2}$, what follows from the Hölder inequality and the fact that

$$
E\left(\bar{q}_{a, N}\right)^{2 n}=\left(2^{2 p} N\right)^{-n} C_{1}\left(1+\sum_{k=1}^{n} \frac{d_{k, n}}{k !} N^{-k}\right),
$$

for some constant $0<C_{1}<\infty$ and the constants $d_{k, n}$ satisfying a bound

$$
\left|d_{k, n}\right|<D^{n}
$$

for some constant $0<D<\infty$. (Note however that as follows from the definition of $\bar{q}_{a, N}$ the polynomial (3.59) contains only the integer powers of $N^{-1}$.)

Using the above information about the expectation $E \bar{q}_{b_{1}, N} \ldots \bar{q}_{b_{j}, N}$ we can represent the sum (3.57) as follows

$$
\Sigma_{2}(K)=\Sigma(K)+\mathcal{O}_{3}(K+1)
$$

with the remainder $\mathcal{O}_{3}(K+1)$ being of order $N^{-(K+2)}$ and the sum

$$
\Sigma(K)=\sum_{k=1}^{K} c_{k} N^{-(k+1)}
$$

with some coefficient $c_{k}$. Using (3.62), (3.55)-(3.57) and (3.52)-(3.53) together with (3.38)-(3.39) and (3.37) we obtain the representation

$$
E f_{N}(\cdot)=-\frac{p}{2 N} \ln (N(1-\beta) / 2 \pi)+\tilde{\Sigma}(K)+\Sigma(K)+R_{K+1} .
$$

This ends the proof of the point $b$ ) and so of Theorem 3.1. 
By this we finished our first treatment of the high temperature case; see Sect. 4 for the result on Borel summability of the expansion.

3.2. Asymptotic Expansions: Low Temperatures. For $\beta>1$, let $x$ denote the positive solution of the equation

$$
x=\beta^{1 / 2} \text { th } \beta^{1 / 2} x .
$$

It follows from the analysis in [13] that the action $S(m)$ given by $(2.4)$ attains its global minimum uniquely at the points $m_{i} \in \mathbb{R}^{d}, i=1, \ldots, 2^{p}$ of the form

$$
m_{i}=\left(0, \ldots, 0, \pm x_{i}, 0, \ldots, 0\right) .
$$

From that it follows that the number $M$ defined by

$$
M^{2} I \equiv D^{2} S\left(m_{i}\right)
$$

(with $I$ the unit matrix and $D^{2} S$ the Hessian) and explicitly given by

$$
M^{2}=1-\beta\left(\operatorname{ch} \beta^{1 / 2} x\right)^{-2}
$$

is strictly positive for $\beta>1$. In this section we prove the following result

Theorem 3.6. Let $\beta>1$. Then for any $K \in \mathbb{N}$ we have the following representations:

a)

$$
\tilde{f}_{N}=-\min _{m \in \mathbb{R}^{p}} S(m)-\frac{p}{2 N} \ln (M \cdot N / 8 \pi)+\tilde{\Sigma}(K)+\tilde{R}_{K+1},
$$

where

$$
\tilde{\Sigma}(K) \equiv \sum_{n=1}^{K} \tilde{c}_{n} N^{-(n+1)}
$$

for some constants $\tilde{c}_{n}, n \in \mathbb{N}$ satisfying the factorial growth condition

$$
\left|\tilde{c}_{n}\right|<\tilde{C}^{n} n !
$$

for some positive constant $\tilde{C}$, and the remainder $\tilde{R}_{K+1}$ is of order $N^{-(K+2)}$ i.e. satisfies

$$
\left|\tilde{R}_{K+1}\right|<\tilde{D}_{K+1} N^{-(K+2)}
$$

for some positive constant $\tilde{D}_{K+1}$.

b)

$$
E f_{N}=-\min _{m \in \mathbb{R}^{d}} S(m)-\frac{p}{2 N} \ln (M \cdot N / 8 \pi)+\Sigma(K)+R_{K+1}
$$

where

$$
\Sigma(K) \equiv \sum_{k=1}^{K} c_{k} N^{-(k+1)}
$$

with some coefficients $c_{k}, k \in \mathbb{N}$ and the remainder term $R_{K+1}$ being of order $N^{-(K+1)}$. 
Proof of Theorem 3.6 a). Let $m_{0}$ be a minimum point of $S(m)$. In a neighborhood $\left\{m:\left|m-m_{0}\right|<\varepsilon\right\}$ we may represent the action $S(m)$ in the following way:

$$
S(m)=S\left(m_{0}\right)+\frac{1}{2} M^{2}\left(m-m_{0}\right)^{2}+U\left(M\left(m-m_{0}\right), m_{0}\right)
$$

with the remainder function

$$
U\left(M\left(m-m_{0}\right), m_{0}\right) \equiv \int_{0}^{1} d t_{1} \ldots \int_{0}^{t_{3}} d t_{4}\left(\frac{d}{d t_{4}}\right)^{2} S\left(m_{0}+t_{4}\left(m-m_{0}\right)\right) .
$$

It will be useful to assume that $\varepsilon>0$ is small enough, so that $S(m)$ is negative and the function

$$
\frac{1}{4} M^{2} \tilde{m}^{2}+U\left(M \cdot \tilde{m}, m_{0}\right)
$$

is convex for $|\tilde{m}| \equiv\left|m-m_{0}\right|<\varepsilon$. Additionally we may and do assume that $\varepsilon>0$ is so chosen that the function $\tilde{m} \mapsto U\left(M \tilde{m}, m_{0}\right)$ has a convergent power series expansion with respect to $\tilde{m}$, whenever $|\tilde{m}|<\varepsilon$. From now on we fix $m$ to be equal to $(x, 0, \ldots, 0)$ and to simplify the notation we write $U(M \tilde{m})$ to denote $U\left(M \tilde{m}, m_{0}\right)$. (This function should not be confused with the function $U$ used in the high temperature case.)

Let $\chi\left(\left|m-m_{i}\right|<\varepsilon\right)$ be the characteristic function of the set $\left\{m:\left|m-m_{i}\right|<\varepsilon\right\}$ and let $\chi_{\varepsilon}^{c}(m)$ denote the characteristic function of the complement of the set $\bigcup_{i=1}, \ldots, 2^{p}\left\{m:\left|m-m_{i}\right|<\varepsilon\right\}$. Using the symmetry of the action $S(m)$ and information about its global minima we have

$$
\begin{aligned}
\tilde{f}_{N} & \equiv \frac{1}{N} \ln \int d m e^{-N S(m)} \\
& =\frac{1}{N} \ln \left\{2^{p} \int d m \chi\left(\left|m-m_{0}\right|<\varepsilon\right) e^{-N S(m)}+\int d m \chi_{\varepsilon}^{c}(m) e^{-N S(m)}\right\} .
\end{aligned}
$$

We shall now prove the following fact.

Lemma 3.7. There are positive constants $C$ and $\gamma$ such that

$$
\frac{\int d m \chi_{\varepsilon}^{c}(m) e^{-N S(m)}}{\int d m \chi\left(\left|m-m_{0}\right|<\varepsilon\right) e^{-N S(m)}}<C e^{-\gamma N} .
$$

Proof of Lemma 3.7. First of all let us note that for any number $A>1$ we have

$$
\begin{aligned}
\int d m \chi\left(\left|m-m_{0}\right|<\varepsilon\right) e^{-N S(m)} & \geqq \int d m \chi\left(\left|m-m_{0}\right|<\varepsilon / A\right) e^{-N S(m)} \\
& \geqq(\varepsilon / A)^{p} \Omega_{p} \exp \left\{-N \max _{\left|m-m_{0}\right|<\varepsilon / A} S(m)\right\},
\end{aligned}
$$

where $\Omega_{p}$ denotes the volume of the unit ball in $\mathbb{R}^{p}$. We may and do assume that $A>1$ is large so that $S(m)<-\alpha<0$ for some $0<\alpha<\infty$ and all $\left|m-m_{0}\right|$ $<\varepsilon / A$. 
On the other hand we have

$$
\begin{gathered}
\int d m \chi_{\varepsilon}^{c}(m) e^{-N S(m) \leqq} \int d m \chi_{\varepsilon}^{c}(m) \chi\left(|m|<R_{\varepsilon}\right) \cdot \exp \left\{-N \min \chi_{\varepsilon}^{c}(m) S(m)\right\} \\
+\int d m \chi\left(|m|>R_{\varepsilon}\right) e^{-\frac{N(1-\varepsilon)}{2} m^{2}},
\end{gathered}
$$

where $R_{\varepsilon} \equiv 2 \beta^{1 / 2} p^{1 / 2} / \varepsilon$ and we have taken into account that for any $a=1, \ldots, 2^{p}$,

i.e.

$$
\frac{\varepsilon}{2} m^{2}-\ln \operatorname{ch} \beta^{1 / 2} m \cdot \xi_{a}>0
$$

$$
S(m)>\frac{1-\varepsilon}{2} m^{2}
$$

for $|m|>R_{\varepsilon}$. By our choice of $\varepsilon$ the function $S(m)$ is strictly convex in each set $\left\{\left|m-m_{i}\right|<\varepsilon\right\}, i=1, \ldots, 2^{p}$. Therefore

$$
-\min \chi_{\varepsilon}^{c}(m) S(m)<-\max _{\left|m-m_{0}\right|<\varepsilon / A} S(m) .
$$

This together with (3.80) and (3.91) implies the lemma.

Now using (3.76) and Lemma 3.7 we see that

$$
\tilde{f}_{N}=\frac{1}{N} \ln 2^{p} \int d m \chi\left(\left|m-m_{0}\right|<\varepsilon\right) e^{-N S(m)}+\mathcal{O}_{0}(N)
$$

with

$$
\left|\mathcal{O}_{0}(N)\right|<C e^{-\gamma N} \text {. }
$$

From (3.85) and the representation (3.73) of the action $S(m)$, by changing the integration variables $m \mapsto M^{-1 / 2} N^{-1 / 2} \tilde{m}+n$, we obtain

$$
\tilde{f}_{N}=S\left(m_{0}\right)-\frac{p}{2 N} \ln (M \cdot N / 8 \pi)+\mathcal{O}_{0}(N)+\frac{1}{N} \ln \rho_{0}\left(\tilde{\chi} e^{-N U\left(N^{-1 / 2} m\right)}\right)
$$

with $\rho_{0}$ being the $(0, \mathrm{I})$-Gaussian measure on $\mathbb{R}^{p}$ and where for simplicity of notation we have set $\tilde{\chi} \equiv \chi\left(|\tilde{m}|<M^{1 / 2} N^{1 / 2} \varepsilon\right)$.

Let us now study in more detail the last term from the rhs of (3.87). Using the property of the Gaussian distribution it is easy to see that

$$
\frac{1}{N} \ln \rho_{0}\left(\tilde{\chi} e^{-N U\left(N^{-1 / 2} m\right)}\right)=\frac{1}{N} \ln \rho_{0}\left(e^{-N U\left(N^{-1 / 2} \tilde{m}\right) \tilde{\chi}}\right)+\mathcal{O}_{1}(N)
$$

with the error

$$
\left|\mathcal{O}_{1}(N)\right| \leqq e^{-\gamma^{\prime} N}
$$

for some positive constant $\gamma^{\prime}$.

By Taylor expansion to the $K^{\text {th }}$ order we get

$$
\begin{aligned}
& \frac{1}{N} \ln \rho_{0}\left(e^{-N U(N-1 / 2 \tilde{m}) \tilde{\chi}}\right) \\
& \quad=\frac{1}{N} \sum_{k=1}^{K} \frac{(-1)^{k}}{k !} \rho_{0}(\underbrace{N U\left(N^{-1 / 2} m\right) \tilde{\chi}, \ldots, N U\left(N^{-1 / 2} \tilde{m}\right) \tilde{\chi}}_{k \text {-times }})+r_{K+1} \\
& \quad \equiv \Sigma_{0}(K)+r_{K+1}
\end{aligned}
$$


with the remainder

$$
r_{K+1} \equiv \int_{0}^{1} d t_{1} \ldots \int_{0}^{t_{K}} d t_{K+1}\left(\frac{d}{d t_{K+1}}\right)^{K+1} \frac{1}{N} \ln \rho_{0}\left(e^{-t_{K+1} N U\left(N^{1 / 2} m\right) \tilde{\chi}}\right) .
$$

Using our choice of $\varepsilon$ so that (3.77) is true and similar considerations as used in the high temperature case it is not difficult to show that $r_{K+1}$ is of order $N^{-(K+2)}$, i.e.

$$
\left|r_{K+1}\right|<C_{0} N^{-(K+2)}
$$

for some positive constant $C_{0}$ (depending on $K$ but independent of $N \in \mathbb{N}$ ).

Now we shall have only to consider the sum $\Sigma_{0}(K)$ from the rhs of (3.90). We may represent it as follows:

$$
\begin{aligned}
\Sigma_{0}(K) & =\frac{1}{N} \sum_{k=1}^{K} \frac{(-1)^{k}}{k !} \rho_{0}(\underbrace{N U\left(N^{-1 / 2} \tilde{m}\right), \ldots, N U\left(N^{-1 / 2} \tilde{m}\right)}_{k \text {-times }})+r_{K+1}^{\prime} \\
& \equiv \Sigma_{1}(K)+r_{K+1}^{\prime}
\end{aligned}
$$

where

$$
\begin{gathered}
r_{K+1}^{\prime} \equiv-\sum_{l=1}^{K}\left(\begin{array}{c}
K \\
l
\end{array}\right) \rho_{0}(\underbrace{N U\left(N^{-1 / 2} \tilde{m}\right) \tilde{\chi}^{c}, \ldots, N U\left(N^{-1 / 2} \tilde{m}\right) \tilde{\chi}^{c}}_{l \text {-times }}, \\
\underbrace{N U\left(N^{-1 / 2} \tilde{m}\right) \tilde{\chi}, \ldots, N U\left(N^{-1 / 2} \tilde{m}\right) \tilde{\chi}}_{K-l \text { times }}) .
\end{gathered}
$$

We have set here $\tilde{\chi}^{c} \equiv 1-\tilde{\chi}$. Using the fact that in each truncated correlation function the characteristic function $1-\tilde{\chi} \equiv \chi\left(|\tilde{m}|>M^{1 / 2} N^{1 / 2} \varepsilon\right)$ appears at least once, together with similar estimates as in (3.13)-(3.17), we get a bound of the form

$$
\left|r_{K+1}^{\prime}\right| \leqq C_{2}(2 K) ! N^{-1} e^{-\gamma^{\prime \prime} N}
$$

for some positive constants $C_{2}$ and $\gamma^{\prime \prime}$, i.e. the remainder $r_{K+1}^{\prime}$ is exponentially small. To finish the proof it is now sufficient to observe that the sum $\Sigma_{1}(K)$ has the same structure as the one on the r.h.s. of (3.8) analysed before. Therefore we can use the similar arguments to get

$$
\Sigma_{1}(K)=\tilde{\Sigma}(K)+r_{K+1}^{\prime \prime}
$$

with the sum $\tilde{\Sigma}(K)$ as in (3.70) and (3.71) and a remainder $r_{K+1}^{\prime \prime}$ of order $N^{-(K+2)}$, i.e. satisfying

$$
\left|r_{K+1}^{\prime \prime}\right|<C_{3}(K) N^{-(K+2)}
$$

for some positive constant $C_{3}(K)$.

By combining (3.88)-(3.97) and (3.87) we obtain

$$
\tilde{f}_{N}=\min _{m \in \mathbb{R}^{p}} S(m)-\frac{p}{2 N} \ln (M \cdot N / 8 \pi)+\tilde{\Sigma}(K)+R_{K+1}
$$

with

$$
R_{K+1} \equiv\left\{\mathcal{O}_{0}(N)+\mathcal{O}_{1}(N)\right\}+\left\{r_{K+1}+r_{K+1}^{\prime}+r_{K+1}^{\prime \prime}\right\}
$$


It follows' from (3.86) and (3.89) together with (3.92), (3.95) and (3.97) that $R_{K+1}$ is of order $N^{-(K+2)}$, i.e. satisfies the inequality (3.72) for some positive constant $\tilde{D}_{K+1}$. This ends the proof of Theorem 3.6 a).

Now we shall prove the second part of Theorem 3.5. Let us mention that several detailed estimates in the proof of this part are very similar to those used in previously considered cases. In order to keep the space necessary for exposition within reasonable bounds, we take advantage of this fact and present below only the essential steps of the proof.

Proof of Theorem 3.5 b). First of all using Proposition 2.6 we have

$$
E f_{N}(\xi)=E \chi_{\delta}(\xi) f_{N}(\xi)+\mathcal{O}_{1}\left(e^{-\delta N^{1 / 2}}\right)
$$

with the characteristic function $\chi_{\delta}(\xi)$ defined in (2.54). As follows from Lemma 3.7 given below, we can and do assume that $0<\delta$ is small enough so that the action $m \mapsto S_{N}(m, \xi) \chi_{\delta}(\xi)$ has exactly $2^{p}$ minima $m_{i}^{\prime}(\xi), i=1, \ldots, 2^{p}$ located at a distance less than $\varepsilon / 2$ from the corresponding minima $m_{i},\left(i=1, \ldots, 2^{p}\right)$, of $S(m)$, with $0<\varepsilon$ being the same as that used in the proof of part a).

Now we can use similar arguments as before (based on an analog of Lemma 3.7), to drop the part of the free energy coming from integration over $m \in\left\{\exists i=1, \ldots, 2^{p}:\left|m-m_{i}^{\prime}(\xi)\right|>\varepsilon\right\}$. In this way we obtain

$$
E f_{N}(\xi)=E \chi_{\delta} \frac{1}{N} \ln \int d m\left(\sum_{i=1}^{2^{p}} \chi\left(m-m_{i}\right) e^{-N S_{N}(m, \xi)}\right)+\mathcal{O}_{2}
$$

with $\mathcal{O}_{2}$ being exponentially small, i.e.

$$
\mathcal{O}_{2} \equiv \mathcal{O}_{1}\left(e^{-\delta N^{1 / 2}}\right)+\mathcal{O}_{2}\left(e^{-\gamma_{1} N}\right)
$$

for some constant $0<\gamma_{1}<\infty$ and

$$
\chi\left(m-m_{i}\right) \equiv \chi\left(\left(m-m_{i}\right)<\frac{\varepsilon}{2}\right) .
$$

Applying again Proposition 2.6 we may represent the rhs of (3.100) as follows:

$$
E f_{N}(\xi)=-S_{0}+E \frac{1}{N} \ln \sum_{i=1}^{2^{p}} \int d m \chi\left(m-m_{i}\right) e^{-N\left(S_{N}(m, \xi)-S_{0}\right)}+\mathcal{O}_{3},
$$

where we set

$$
S_{0} \equiv \min _{m \in \mathbb{R}^{p}} S(m)
$$

and $\mathcal{O}_{3}$ is some exponentially small remainder. Let us define now for $i=1, \ldots, 2^{p}$,

$$
U_{i}(M \tilde{m}) \equiv U\left(M \tilde{m}, m_{i}, \xi\right)=S_{N}\left(M \tilde{m}+m_{i}, \xi\right)-\frac{1}{2} M^{2} \tilde{m}^{2}-S_{0} .
$$

Using this notation and arguments similar to those used in the proof of part a) we obtain

$$
E f_{N}(\xi)=S_{0}-\frac{p}{2 N} \ln (M \cdot N / 8 \pi)+E \frac{1}{N} \ln \frac{1}{2^{p}} \sum_{i=1}^{2^{p}} \rho_{0}\left(\chi(\tilde{m}) e^{-N U_{i}\left(N^{-1 / 2} \tilde{m}\right)}\right)+\mathcal{O}_{3}
$$


with $\rho_{0}$ being the $(0, I)$-Gaussian measure on $\mathbb{R}^{p}$. This can be rewritten in the following form:

$$
E f_{N}(\xi)=S_{0}-\frac{p}{2 N} \ln (M \cdot N / 8 \pi)+E \frac{1}{N} \ln \frac{1}{2^{p}} \sum_{i=1}^{2^{p}} \rho_{0}\left(e^{-N \chi(\tilde{m}) U_{i}\left(N^{-1 / 2} \tilde{m}\right)}\right)+\mathcal{O}_{4}
$$

with an exponentially small remainder $\dot{\mathcal{O}}_{4}$.

Now we introduce an interpolating parameter $t \in[0,1]$ into the exponential in the Gaussian expectation and generate the Taylor expansion to a finite order $K$. The estimate of the remainder of this expansion can be done easily in a similar way as in the proof of part a). Then omitting the cut-off function $\chi(\tilde{m})$ in particular terms of this expansion we get the representation

$$
\begin{aligned}
& E \frac{1}{N} \ln \frac{1}{2^{p}} \sum_{i=1}^{2^{p}} \rho_{0}\left(e^{-N \chi(\tilde{m}) U_{i}\left(N^{-1 / 2} \tilde{m}\right)}\right) \\
& =\frac{1}{N} \sum_{k=1}^{K} \frac{(-1)^{k}}{k !} E \mu \underbrace{\left(N U_{0}\left(N^{-1 / 2} \cdot\right), \ldots, N U_{0}\left(N^{-1 / 2} \cdot\right)\right)}_{k \text {-times }}+r\left(N^{-(K+2)}\right),
\end{aligned}
$$

where for simplicity of notation we introduced the following probability measure:

$$
\mu f .(\tilde{m}) \equiv \frac{1}{2^{p}} \sum_{i=1}^{2^{p}} \rho_{0} f_{i}(\tilde{m}) .
$$

Now we expand each function $U_{i}\left(N^{-1 / 2} \tilde{m}\right)$, with respect to $N^{-1 / 2} \tilde{m}$, up to order $K$. By reorganizing this expansion and similar, but quite lengthy, analysis as in the proof of part a), one arrives at the desired asymptotic expansion in the present case. (Let us note that the additional averaging with respect to the discrete measure is a minor complication, due to the fact that the coefficients of the Taylor expansion of the function $U_{i}\left(N^{-1 / 2} \tilde{m}\right)$ are in fact independent of $i=1, \ldots, 2^{p}$.)

To finish our considerations we shall now have only to prove a lemma providing the information about minima of the random action used at the beginning of the proof of Theorem $3.6 \mathrm{~b}$ ).

Lemma 3.8. Let $\delta>0$ and let

$$
A_{\delta} \equiv\left\{\xi:\left|q_{a, N}(\xi)-\frac{1}{2^{p}}\right|<\delta, a=1, \ldots, 2^{p}\right\} .
$$

For any minimum $m_{i}$ of $S(m)$ and any $\varepsilon>0$, there is $\delta>0$ such that, whenever $\xi \in A_{\delta}$, the function

$$
m \mapsto S_{N}(m, \xi)
$$

has a unique minimum $m_{i}(\xi)$ such that

$$
\left|m_{i}-m_{i}(\xi)\right|<\varepsilon .
$$

Additionally if $\beta<1$ and $\delta \leqq N^{-1 / 2}$ then for any $\xi \in A_{\delta}$, the corresponding action $S_{N}(m, \xi)$ has a unique minimum at zero and is strictly convex for all $N \geqq N_{0}$, with some $N_{0} \in \mathbb{N}$ sufficiently large. (The same is true if one replaces $S_{N}(m, \xi)$ by $N S_{N}\left(N^{-1 / 2} m, \xi\right)$.) 
Proof. We have easily from the definition of $S_{N}$ that $\frac{\partial}{\partial m^{\alpha}} S_{N}(m, \xi)$ exists for any $\alpha$ and

$$
\frac{\partial}{\partial m^{\alpha}} S_{N}(m, \xi)=m^{\alpha}-\sum_{a=1}^{2^{p}} q_{a, N}(\xi) \beta^{1 / 2} \xi_{a}^{\alpha} \tanh \left(\beta^{1 / 2} m \xi_{a}\right)
$$

and

$$
\frac{\partial^{2}}{\partial m^{\alpha_{1}} \partial m^{\alpha_{2}}} S_{N}(m, \xi)=\delta^{\alpha_{1}, \alpha_{2}}-\sum_{a=1}^{2^{p}} q_{a, N}(\xi) \beta\left[\cosh \left(\beta^{1 / 2} m \xi_{a}\right)\right]^{-2} \xi_{a}^{\alpha_{1}} \xi_{a}^{\alpha_{2}}
$$

We see easily that (3.112) and (3.113) are continuous in $m$, and we have:

$$
\begin{aligned}
& \left|\frac{\partial}{\partial m^{\alpha}} S_{N}(m, \xi)-\frac{\partial}{\partial m^{\prime \alpha}} S_{N}\left(m^{\prime}, \xi\right)\right| \\
& \quad \leqq\left|m^{\alpha}-\left(m^{\prime}\right)^{\alpha}\right|+\sum_{a=1}^{2^{p}} q_{a, N}(\xi) \beta\left[\cosh \left(\beta^{1 / 2} m \xi_{a}\right)\right]^{-2}\left|\left(m-m^{\prime}\right) \cdot \xi_{a}\right| \\
& \quad \leqq(1+\beta) \sum_{\alpha=1}^{p}\left|m^{\alpha}-\left(m^{\prime}\right)^{\alpha}\right|
\end{aligned}
$$

and

$$
\left|\frac{\partial^{2}}{\partial m^{\alpha_{1}} \partial m^{\alpha_{2}}} S_{N}(m, \xi)-\frac{\partial^{2}}{\partial m^{\prime \alpha_{1}} \partial m^{\prime \alpha_{2}}} S_{N}\left(m^{\prime}, \xi\right)\right| \leqq 2 \beta^{3 / 2} \sum_{\alpha=1}^{p}\left|m^{\alpha}-m^{\prime \alpha}\right| .
$$

For fixed $m$, we also see that both

$$
\frac{\partial}{\partial m^{\alpha}} S_{N}(m, \xi)
$$

and

$$
\frac{\partial^{2}}{\partial m^{\alpha_{1}} \partial m^{\alpha_{2}}} S_{N}(m, \xi)
$$

are continuous, uniformly in $m$, with respect to $q_{a, N}(\xi)$.

Now let $m_{i}$ be a point of minimum for $S(m)$ i.e.:

$$
\frac{\partial}{\partial m^{\alpha}} S_{N}\left(m_{i}\right)=0
$$

and the matrix:

$$
\left(\left(\frac{\partial^{2}}{\partial m^{\alpha_{1}} \partial m^{\alpha_{2}}} S\left(m_{i}\right)\right)\right)
$$

is positive definite. By the above continuity, for any $\varepsilon>0$, there exists $\delta>0$ such that for each $\xi \in A_{\delta}$ there exists a unique point $m_{i}(\xi)$ satisfying

$$
\begin{gathered}
\left|m_{i}-m_{i}(\xi)\right|<\delta, \\
\frac{\partial}{\partial m^{\alpha}} S_{N}\left(m_{i}(\xi), \xi\right)=0,
\end{gathered}
$$


and the matrix

$$
\left(\left(\frac{\partial^{2}}{\partial m^{\alpha_{1}} \partial m^{\alpha_{2}}} S\left(m_{i}(\xi), \xi\right)\right)\right)
$$

is positive definite, i.e. $m_{i}(\xi)$ is a minimum.

To show the last statement we note that

$$
S_{N}(m, \xi)=S(m)+\sum_{a=1}^{2^{p}} \bar{q}_{a, N}(\xi) \ln \cosh \left(\beta^{1 / 2} m \cdot \xi_{a}\right) .
$$

Hence clearly $m=0$ is the critical point of the action. Additionally we have $\frac{\partial^{2}}{\partial m^{\alpha_{1}} \partial m^{\alpha_{2}}} S_{N}(m, \xi)=\frac{\partial^{2}}{\partial m^{\alpha_{1}} \partial m^{\alpha_{2}}} S(m)+\sum_{a=1}^{2^{p}} \bar{q}_{a, N}(\xi) \beta\left[\cosh \left(\beta^{1 / 2} m \xi_{a}\right)\right]^{-2} \xi_{a}^{\alpha_{1}} \xi_{a}^{\alpha_{2}}$.

By our assumption about $\bar{q}_{a, N}(\xi)$ the second term on the rhs of (3.119) can be made arbitrarily small (uniformly in $m$ ) by taking $N \geqq N_{0}$, for some $N_{0}$ sufficiently large. Since for $\beta<1$ the action $S(m)$ is strictly convex, the last statement of the lemma follows.

\section{Borel Summability}

In this section we shall study the partition function $Z_{N}$ given by (1.9) and show that it has a Borel summable expansion in powers of $\lambda \equiv 1 / N$ as $N \rightarrow \infty$. To do this we split up the integration with respect to the variable $m \in \mathbb{R}^{p}$ in (1.9) into an integration over the set $A_{a} \equiv\left\{m \in \mathbb{R}^{p}|| m \mid \leqq 2 \frac{\sqrt{p \beta}}{a}\right\}$, for some $0<a<1$ and its complement $A_{a}^{c}$.

From (1.9), (2.4), (2.7) it suffices to study

$$
\int_{\boldsymbol{A}_{a}} d m \exp (-N S(m)) \equiv Z_{N, a}
$$

and

$$
\int_{A_{a}^{c}} d m \exp (-N S(m)) \equiv Z_{N, a}^{c}
$$

Lemma 4.1. For any $0<a<1$,

with

$$
Z_{N, a}^{c} \leqq c_{a} \exp \left[-(1-a) \frac{2 p}{a^{2}} \beta(N-1)\right]
$$

$$
c_{a} \equiv \int_{\mathbb{R}^{p}} d m \exp \left[-\frac{1}{2}(1-a) m^{2}\right]<\infty .
$$

Proof. For $m \in A_{a}^{c}$ we have, recalling the definition (2.4) of $S(m)$ :

$$
S(m)=\frac{1}{2} m^{2}-E \ln \operatorname{ch}\left(\beta^{1 / 2} m \xi\right) \geqq \frac{1}{2} m^{2}-\sqrt{p \beta}|m| \geqq \frac{1}{2}(1-a) m^{2} .
$$


Inserting this into (4.1) we get

$$
\begin{aligned}
Z_{N, a}^{c} & =\int_{\boldsymbol{A}_{\boldsymbol{a}}^{c}} d m e^{-(N-1) S(m)} e^{-S(m)} \\
& \leqq e^{-(1-a) \frac{2 p}{a^{2}} \beta(N-1)} \int_{A_{a}^{c}} d m e^{-S(m)} \\
& \leqq e^{-(1-a) \frac{2 p}{a^{2}} \beta(N-1)} c_{a},
\end{aligned}
$$

where in the last but one inequality we have used the definition of $A_{a}^{c}$ and in the last inequality the definition of $c_{a}$.

This lemma then shows that the contribution $Z_{N, a}^{c}$ to $Z_{N}$, coming from the integration over $A_{a}^{c}$, is exponentially small in $N$, for $N \rightarrow \infty$.

The contribution coming from the integration over $A_{a}$ is controlled by the following

Lemma 4.2. For any $0<\beta<1$ and any $0<a<1$ we have, for all $|\lambda|<$ $\min \left(\frac{a}{4 \beta^{2} p^{2}}, \rho\right)$,

$$
Z_{N, a}=\sum_{j=0}^{k}(\beta \lambda)^{j} \int_{A_{a}} e^{-\frac{1}{2} m^{2}} \tilde{c}_{j}(m) d m+\int_{A_{a}} e^{-\frac{1}{2} m^{2}} R_{k}(m) d m,
$$

with

$$
\begin{aligned}
\tilde{c}_{j}(m) & \equiv \sum_{n=0}^{\infty} \frac{\beta^{n}}{n !} \sum_{m_{1}, \ldots, m_{n} \in \mathbb{N}_{0}} \prod_{i=1}^{n}\left[E(m \cdot \xi)^{2\left(m_{i}+1\right)}\right] C_{m_{i}+1}, \\
\sum_{i=1}^{n} m_{i} & =j \\
C_{j+1} & \equiv \sum_{l=1}^{j+1} \frac{(-1)^{l}}{l} \sum_{K_{1}, K_{i \geqq 1} K_{l}} \prod_{i=1}^{l} \frac{1}{\left(2 K_{i}\right) !}, \quad i \in \mathbb{N}, \\
\sum_{i=1}^{l} K_{i} & =j+1, \\
C_{1} & \equiv 1, \\
R_{k}(m) & \equiv \exp \left[\frac{1}{\lambda} E \ln \operatorname{ch}(\sqrt{\beta \lambda} m \cdot \xi)\right]-\sum_{i=0}^{k}(\beta \lambda)^{j} \tilde{c}_{j}(m) .
\end{aligned}
$$

The following bounds hold for all $j$

$$
\left|\int_{\boldsymbol{A}_{\boldsymbol{a}}} d m e^{-1 / 2 m^{2}} \tilde{c}_{j}(m) d m\right| \leqq C 2^{-2 j} p^{j} \tilde{C}_{j} e^{\frac{\beta p}{8}(j+1)},
$$

for some constants $C, \tilde{C}_{j}$ (depending on a), and

$$
\left|\int_{A_{a}} d m e^{-1 / 2 m^{2}} R_{k}(m)\right| \leqq C^{\prime k}|\lambda|^{k} k !
$$

for some $C^{\prime}>0$. 
Proof. For $0<\beta<1$ we have, by $(2.25), \frac{1}{2}(1-\beta) m^{2} \leqq S(m)$.

Let $\lambda=1 / N$, be in $\mathbb{C}$. Then, for $\operatorname{Re} N>0$, for any measurable $B \subset \mathbb{R}^{p}$ :

$$
\begin{aligned}
\int_{B} d m \exp [-N S(m)] & \leqq \int_{B} d m|\exp (-N S(m))| \\
& =\int_{B} d m\left|e^{\operatorname{Re} N S(m)}\right| \\
& \leqq \int_{B} d m e^{-(\operatorname{Re} N) \frac{1}{2}(1-\beta) m^{2}} \\
& \leqq \int_{\mathbb{R}^{p}} d m e^{-(\operatorname{Re} N) \frac{1}{2}(1-\beta) m^{2}}<\infty
\end{aligned}
$$

For $m \in A_{a}$ we have

$$
\beta|\lambda||m \cdot \xi|^{2} \leqq \frac{4}{a^{2}} \beta^{2}|\lambda| p^{2}<1
$$

provided

$$
|\lambda|<\frac{a^{2}}{4 \beta^{2} p^{2}}
$$

$\operatorname{lnch} z$ is analytic in $z$ for $z$ such that

$$
|\operatorname{ch} z-1|<1 \text {. }
$$

In particular this is the case when

$$
|z|<\rho<1
$$

for some $\rho>0$.

With $z \equiv \sqrt{\beta \lambda}(m \cdot \xi)$ this is satisfied, together with (4.3), when

$$
|\lambda|<\min \left(\frac{a^{2}}{4 \beta^{2} p^{2}}, \rho\right), \quad m \in A_{a} .
$$

An easy direct expansion in power series of the functions $1 n$ and ch yields

$$
\operatorname{lnch} \sqrt{\beta \lambda}(m \cdot \xi)=(\beta \lambda) \sum_{j=0}^{\infty}(\beta \lambda)^{j}(m \cdot \xi)^{2(j+1)} C_{j+1},
$$

with $C_{j+1}, C_{1}$ as given in the lemma. The series is convergent for $|\lambda|$ as above. An easy estimate yields

$$
\left|C_{j+1}\right| \leqq C \frac{2^{-2(j+1)}}{2(j+1)}
$$

for some $C>0$. (Incidentally by the general Cauchy inequalities one knows already the bound $\left|C_{j+1}\right| \leqq \tilde{\rho}^{j} \sup |\ln \operatorname{ch} \sqrt{\beta \lambda}(m \cdot \xi)|<\infty$, the sup being taken over $\sqrt{\beta \lambda} m \cdot \xi=\tilde{\rho}, m \in A_{a}$, for any $\tilde{\rho}<\rho$.)

Using that exp is an entire function we then get, for $m, \lambda$ as above, the absolutely convergent expansion

$$
\exp \left(\frac{1}{\lambda} E \ln \operatorname{ch}(\sqrt{\beta \lambda} m \cdot \xi)\right)=\sum_{n=0}^{\infty} \frac{\beta^{n}}{n !}\left[\sum_{j=0}^{\infty}(\beta \lambda)^{j}(m \cdot \xi)^{2(j+1)} C_{j+1}\right]^{n}
$$


Interchanging the $j$ and $n$ summation we obtain

$$
\exp \left(\frac{1}{\lambda} E \ln \operatorname{ch}(\sqrt{\beta \lambda} m \cdot \xi)\right)=\sum_{n=0}^{k}(\beta \lambda)^{j} \tilde{c}_{j}(m)+R_{k}(m)
$$

with $\tilde{c}_{j}(m), R_{k}(m)$ as in the statement of the lemma.

Call $\tilde{c}_{j, M}(m)$ the series obtained by truncating $\tilde{c}_{j}(m)$, setting its terms equal to 0 after the $M^{\text {th }}$ order in $\beta$, so that $\lim _{M \rightarrow \infty} \tilde{c}_{j, M}(m)=\tilde{c}_{j}(m)$. We have the bounds

$$
\begin{aligned}
\left|\tilde{c}_{j, M}(m)\right| & \leqq C \sum_{n=0}^{M} \frac{\beta^{n}}{n !} \sum_{\substack{m_{1}, \ldots \ldots m_{i} \geqq 0 \\
m_{i}}} 2^{-2(j+n)}\left(p m^{2}\right)^{j+n} \prod_{i=1}^{n} \frac{1}{2\left(m_{i}+1\right)}, \\
\sum_{i=1}^{n} m_{i} & =j .
\end{aligned}
$$

Let

$$
F\left(m^{2}, j, n\right) \equiv \exp \left[-\frac{m^{2}}{2}\right]\left(m^{2}\right)^{j+n},
$$

defined for $m^{2} \in \mathbb{R}, 0 \leqq n \leqq M, j \in \mathbb{N}$. This function is monotone in $m^{2}$ and its maximum is given by the $m$-value $m_{\max }$ such that

$$
0<m^{2}{ }_{\text {max }}=2(j+n) .
$$

Hence

$$
F\left(m^{2}, j, n\right) \leqq \exp [-(j+n)][2(j+n)]^{j+n}
$$

Since for $m \in A_{a}$ we have $m^{2}<2 K_{\beta} \equiv \frac{4 p \beta}{a^{2}}$ we distinguish the cases

$$
j+n<K_{\beta} \text {, i.e. } m^{2}{ }_{\max }<2 K_{\beta} \text {, }
$$

and

$$
j+n \geqq K_{\beta} \text {, i.e. } m^{2}{ }_{\max } \geqq 2 K_{\beta} \text {. }
$$

In the case (4.22) we have from (4.21), for $m \in A_{a}$,

$$
F\left(m^{2}, j, n\right) \leqq \exp \left[-K_{\beta}\right]\left(2 K_{\beta}\right)^{2 K_{\beta}} .
$$

In the case (4.23) we have from (2.21), for $m \in A_{a}$, that the bound (4.21) can be replaced by the monotonicity of $m^{2} \rightarrow F\left(m^{2}, j, n\right)$ for large $m^{2}$ and the fact that $m^{2}{ }_{\text {max }} \geqq 2 K_{\beta}$ implies $m_{\max } \notin A_{a}$, by

$$
F\left(m^{2}, j, n\right) \leqq \exp \left(-K_{\beta}\right)\left(2 K_{\beta}\right)^{j+n},
$$

for $m \in A_{a}$. Hence

$$
\int_{\boldsymbol{A}_{a}} d m e^{-\frac{1}{2} m^{2}} F\left(m^{2}, j, n\right) \leqq C \exp \left[-K_{\beta}\right]\left(2 K_{\beta}\right)^{2 K_{\beta}},
$$

in case (4.22), for some $C>0$, and

$$
\int d m e^{-\frac{1}{2} m^{2}} F\left(m^{2}, j, n\right) \leqq C \exp \left[-K_{\beta}\right]\left(2 K_{\beta}\right)^{j+n},
$$


in case (4.23). Inserting these estimates into

$$
\int_{\boldsymbol{A}_{\boldsymbol{a}}} d m e^{-\frac{1}{2} m^{2}} \tilde{c}_{j, M}(m)
$$

and exploiting

$$
\sum_{n=0}^{M}\left(\frac{\beta p}{8}\right)^{n} \frac{1}{n !} \sum_{\substack{m_{i}>0 \\ \sum m_{i}=j}} 1 \leqq \sum_{n=0}^{\infty}\left(\frac{\beta p}{8}\right)^{n} \frac{1}{n !}(j+1)^{n}=e^{\frac{\beta p}{8}(j+1)}
$$

we get the bound

$$
\int_{\boldsymbol{A}_{a}} d m e^{-\frac{1}{2} m^{2}} \tilde{C}_{j, M}(m) \leqq C 2^{-2 j} p^{j} \tilde{C}_{j} e^{\frac{\beta \gamma p}{8}(j+1)}
$$

with

$$
\begin{aligned}
\tilde{C}_{j} & \equiv \max \left[\left(2 K_{\beta}\right)^{K_{\beta}},\left(2 K_{\beta}\right)^{j}\right] \exp \left(-K_{\beta}\right), \\
\gamma & =\max \left(1,2 K_{\beta}\right) .
\end{aligned}
$$

By dominated convergence the same bound holds with $\tilde{c}_{j, M}$ replaced by $\tilde{c}_{j}$, which proves the last but one estimate in the lemma. The estimate on the remainder is easily obtained by using Taylor's formula and the fact that integrations are on the region $A_{a}$ of finite volume.

From Lemma 4.1, Lemma 4.2, together with the fact that by the argument given at the beginning of the proof of Lemma 4.2,

$$
\int d m \exp (-N S(m))
$$

is analytic in $\lambda=\frac{1}{N}$ in the region $\operatorname{Re} \frac{1}{\lambda}>\frac{1}{R}$ for any $R>0$, such that $\min \left(\frac{a^{2}}{4 \beta^{2} p^{2}}, \rho\right)<R, \rho<1$, and we can use a Theorem of Nevanlinna, see e.g. [14], we have then, the following

Theorem 4.3. Let $Z_{N}$ be as in Theorem 3.1. Then the asymptotic expansion of Theorem 3.1 in powers of $\frac{1}{N}$ is Borel summable.

\section{Appendix}

Let $E_{0}$ be a probability measure on $\{-1,+1\}^{p}$ defined as the product of uniform probability measures on $\{-1,+1\}$. Let $\xi \equiv\left(\xi^{i}\right)_{i \in \mathbb{N}}$, with $\xi^{i} \in\{-1,+1\}^{p}$ being independent identically distributed (i.i.d.) random variables. The corresponding probability measure on $\left(\{-1,+1\}^{p}\right)^{\mathbf{N}}$ is denoted by $E$ and equal to the product of the measures $E_{0}$.

For $N \in \mathbb{N}$ and a fixed vector $\xi_{0} \in\{-1,+1\}^{p}, a=1, \ldots, 2^{p}$ we set

$$
q_{a, N}(\xi) \equiv \frac{1}{N} \sum_{i=1}^{N} \delta_{\xi^{i}, \xi_{a}}
$$


and

$$
\bar{q}_{a, N}(\xi) \equiv q_{a, N}(\xi)-\frac{1}{2^{p}}
$$

With the notation introduced above we have the following lemma.

Lemma A.1. For any $t \in \mathbb{R}, N \in \mathbb{N}$ and $a=1, \ldots, 2^{p}$ we have

$$
\begin{aligned}
E e^{t \bar{q}_{a}, N} & =e^{-\frac{t}{2^{p}}}\left(1+\frac{1}{2^{p}}\left(e^{t / N}-1\right)\right)^{N} \\
& \leqq \exp \left\{\frac{1}{2^{p}} \sum_{n \geqq 2} \frac{t^{n}}{n !} N^{-n+1}\right\} .
\end{aligned}
$$

Proof. The formula for $E e^{t \bar{q}_{a}, N}$ in (A.3) follows from definitions (A.1)-(A.2) by simple explicit calculations. To get the upper bound we use the fact that

$$
\left(1+\frac{1}{2^{p}}\left(e^{t / N}-1\right)\right)^{N} \leqq \exp \left\{\frac{1}{2^{p}} N\left(e^{t / N}-1\right)\right\} .
$$

For $\delta>0$, let $\chi_{\delta, N}$ denote a characteristic function of the event $\left\{\xi:\left|\bar{q}_{a, N}(\xi)\right|<\right.$ $\left.\delta, \forall a=1, \ldots, 2^{p}\right\}$.

Using Lemma A.1 we get

Lemma A.2. There is a constant $0<C_{1}<\infty$ independent of $\delta>0$ and $N \in \mathbb{N}$ such that

$$
E\left(1-\chi_{\delta, N}\right) \leqq C_{1} e^{-\delta N^{1 / 2}}
$$

Proof. We have

$$
E\left(1-\chi_{\delta, N}\right) \leqq \sum_{a=1}^{2 p} E\left\{\left|\bar{q}_{a, N}\right|>\delta\right\} .
$$

Using the following inequality for $t>0$,

$$
\begin{aligned}
E\left\{\left|\bar{q}_{a, N}\right|>\delta\right\} & =E\left\{e^{t\left|\bar{q}_{a, N}\right|}>e^{t \delta}\right\} \\
& \leqq E\left\{e^{t \bar{q}_{a}, N}+e^{-t \bar{q}_{a}, N}>e^{t \delta}\right\}
\end{aligned}
$$

together with Tschebyschev inequality and Lemma A.1 we get

$$
\begin{aligned}
E\left\{\left|\bar{q}_{a, N}\right|>\delta\right\} & \leqq e^{-t \delta} E\left(e^{t \bar{q}_{a}, N}+e^{-t \bar{q}_{a}, N}\right) \\
& \leqq 2 e^{-t \delta} \exp \left\{\frac{1}{2^{p}} \sum_{n \geqq 2} \frac{t^{n}}{n !} N^{-n+1}\right\} .
\end{aligned}
$$

Now we use (A.8) with $t=N^{1 / 2}$ to bound the rhs of (A.6). By this we get (A.5) with

$$
C_{1}=2^{p+1} \exp \left\{\frac{1}{2^{p}}(e-1)\right\} \text {. }
$$


Remark. By optimalisation with respect to $t$ on the rhs of (A.8) one can get an improved bound (A.5) with $N$ instead $N^{1 / 2}$ in the exponent.

\section{Lemma A.3.}

a)

$$
E\left|\bar{q}_{a, N}\right| \leqq\left(\frac{1}{2^{p}}-\frac{1}{2^{2 p}}\right) \frac{1}{N^{1 / 2}} .
$$

b) For any $0<C_{2}<\infty$, there is a constant $0<C_{3}<\infty$ independent of $a \in$ $\left\{1, \ldots, 2^{p}\right\}, N \in \mathbb{N}$ and $n \in \mathbb{N}$ such that

$$
E\left|\bar{q}_{a, N}\right|^{n} \leqq C_{3} n !\left(C_{2} N^{1 / 2}\right)^{-n} .
$$

c) We have with some positive constant $0<C_{4}<\infty$,

$$
E\left(\bar{q}_{a, N}\right)^{2 n}=N^{-n} C_{4}^{n}\left(1+\sum_{k=1}^{n-1} \frac{d_{k, n}}{k !} N^{-k}\right),
$$

where the constants $d_{k, n}$ satisfy

$$
0 \leqq\left|d_{k, n}\right| \leqq D^{n}
$$

with some $0<D<\infty$.

Proof. Using the definition (A.1) of $q_{a, N}$ we get

$$
\begin{aligned}
E\left[\left(q_{a, N}(\cdot)-\frac{1}{2^{p}}\right)^{2}\right] & =E\left(\frac{1}{N^{2}} \sum_{i, j \in(1 \ldots N)} \delta_{\xi_{i}, \xi_{a}} \delta_{\xi_{j}, \xi_{a}}-2 \frac{1}{2^{p} N} \sum_{i=1}^{N} \delta_{\xi^{i}, \xi_{a}}+\frac{1}{2^{2 p}}\right) \\
& =E\left(\frac{1}{N^{2}} \sum_{i, j=1}^{N} \delta_{\xi^{2}, \xi_{a}} \delta_{\xi^{j}, \xi_{a}}-\frac{1}{2^{2 p}}\right) .
\end{aligned}
$$

But using the independence of $\xi_{i}, \xi_{j}$ for $i \neq j$ and $\left(\delta_{\xi_{i}, \xi_{a}}\right)^{2}=\delta_{\xi_{i}, \xi_{a}}$ we get:

$$
\begin{aligned}
E\left(\frac{1}{N^{2}} \sum_{i, j=1}^{N} \delta_{\xi^{i}, \xi_{a}} \delta_{\xi^{j}, \xi_{a}}\right) & =E\left(\frac{1}{N^{2}} \sum_{i \neq j}^{N} \delta_{\xi^{i}, \xi_{a}} \delta_{\xi^{j}, \xi_{a}}\right)+E\left(\frac{1}{N^{2}} \sum_{i=j}^{N}\left(\delta_{\xi^{i}, \xi_{a}}\right)^{2}\right) \\
& =\frac{1}{N^{2}} \sum_{i \neq j}^{N} E\left(\delta_{\xi^{i}, \xi_{a}}\right) E\left(\delta_{\xi^{j}, \xi_{a}}\right)+\frac{1}{N^{2}} \sum_{i=j}^{N} E\left(\delta_{\xi^{i}, \xi_{a}}\right) \\
& =\frac{1}{N^{2}} N(N-1) \frac{1}{2^{2 p}}+\frac{1}{N} \frac{1}{2^{p}}
\end{aligned}
$$

Using (A.14) and (A.15) we have:

$$
E\left[\left(q_{a, N}(\cdot)-\frac{1}{2^{p}}\right)^{2}\right]=\frac{1}{N}\left(\frac{1}{2^{p}}-\frac{1}{2^{2 p}}\right) .
$$

From this and the Schwarz inequality, Lemma A.3a is obtained.

Let us now prove $\mathrm{b}$ ). We have for any positive $t$,

$$
E\left(\bar{q}_{a, N}\right)^{2 n} \leqq(2 n) ! t^{-2 n} E \operatorname{ch}\left(t \bar{q}_{a, N}\right) .
$$


Choosing $t=\left(4 C_{2} N\right)^{1 / 2}$ and using Lemma A.1 we get

$$
E\left(\bar{q}_{a, N}\right)^{2 n} \leqq(2 n) !\left(4 C_{2}\right)^{-n} N^{-n} \exp \left\{\frac{1}{2^{p}} \sum_{k \geqq 2} \frac{\left(4 C_{2}\right)^{\frac{k}{2}}}{k !} N^{-\frac{k}{2}+1}\right\} .
$$

Hence, by application of Hölder inequality, we obtain

$$
E\left|\bar{q}_{a, N}\right|^{n} \leqq C_{3} n !\left(C_{2} N\right)^{-(1 / 2) n}
$$

with

$$
C_{3}=\exp \left\{\frac{1}{2^{p+1}} \sum_{k \geqq 2} \frac{\left(4 C_{2}\right)^{\frac{k}{2}}}{k !}\right\} .
$$

This ends the proof of part b).

To show c) we use the explicit formula for $E e^{t \bar{q}_{a}, N}$ given in Lemma A.1. We have for $t \in \mathbb{R},|t|$ sufficiently small

$$
E e^{t \bar{q}_{a}, N}=e^{-\frac{t}{2^{p}}}\left(1+\frac{1}{2^{p}}\left(e^{t / N}-1\right)\right)^{N} \equiv \exp f\left(2^{-p} N^{-1 / 2} t\right),
$$

where

$$
f\left(2^{-p} N^{-1 / 2} t\right) \equiv \frac{1}{2^{p}} \sum_{k \geqq 2}^{\infty} \frac{t^{k}}{k !} N^{-k+1}+N \sum_{m \geqq 2}^{\infty} \frac{(-1)^{m}}{m !}\left(\frac{1}{2^{p}}\left(e^{t / N}-1\right)\right)^{m} .
$$

Using (A.21)-(A.22) we have

$$
E\left(\bar{q}_{a, N}\right)^{2 n}=\frac{d^{2 n}}{(d t)^{2 n}} E e^{t \bar{q}_{a}, N}=\left.\left(2^{2 p} N\right)^{-n} \frac{d^{2 n}}{(d z)^{2 n}} \exp f(z)\right|_{z=0} .
$$

We expand the exponential $\exp f(z)$ in powers of $f(z)$ and use the formula

$$
\left.\frac{d^{2 n}}{(d z)^{2 n}} f^{k}(z)\right|_{z=0}=\left.\sum_{\substack{j_{1}, \ldots, j_{k} \geqq 2 \\ \sum_{i=1}^{k} j_{i}=2 n}} \frac{(2 n) !}{j_{1} !, \ldots, j_{k} !} \prod_{i=1}^{k} \frac{d^{j_{i}}}{(d z)^{j_{i}}} f(z)\right|_{z=0} .
$$

Now using (A.22) we get for $j \geqq 2$,

$$
\left.\left.\frac{d^{j}}{(d z)^{j}} f(z)\right|_{z=0}=2^{p j-1} N^{-1 / 2 j+1}+\frac{d^{j}}{d z^{j}} N \sum_{m \geqq 2} \frac{(-1)^{m}}{m !}\left(\frac{1}{2^{p}} e^{2^{p}{ }^{2} / N^{1 / 2}}-1\right)\right)\left.^{m}\right|_{z=0} .
$$

Since

$$
\begin{aligned}
& \left.\frac{d^{j}}{(d z)^{j}}\left(e^{2^{p} / N^{1 / 2}}-1\right)^{m}\right|_{z=0}
\end{aligned}
$$

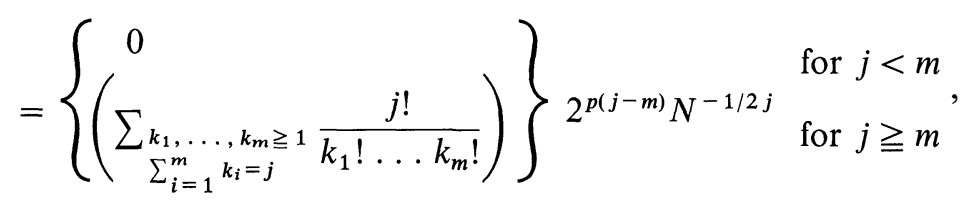


so we get for $j \geqq 2$,

$$
\begin{aligned}
\left.\frac{d^{j}}{(d z)^{j}} f(z)\right|_{z=0}= & 2^{p j-1} N^{-1 / 2 j+1} \\
& +N \sum_{m=2}^{j} \frac{(-1)^{m}}{m !}\left(\sum_{\substack{k_{1}, \ldots, k_{m} \geqq 1 \\
\sum_{i=1}^{m} k_{i}=j}} \frac{j !}{k_{1} ! \ldots k_{m} !}\right) 2^{p(j-m)} N^{-1 / 2 j} \\
= & 2^{p j-1} N^{-1 / 2 j+1}\left\{1+\sum_{m=2}^{j} \frac{(-1)^{m}}{m !}\right. \\
& \left.\times\left(\sum_{\substack{k_{\imath}, \ldots, k_{m} \geqq 1 \\
\sum_{i=1}^{m} k_{i}=j}}^{j ! \ldots k_{m} !}\right) 2^{p m+1}\right\} \\
= & 2^{p j-1} N^{-1 / 2 j+1} a_{j} .
\end{aligned}
$$

Let us note that the sum $a_{j}$ (in the curly bracket on the rhs of (A.27)) is bounded by $2^{j} e^{-\left(2^{-p+1}\right)}$. Inserting (A.27) into (A.24) we obtain

$$
\begin{aligned}
\left.\frac{d^{2 n}}{(d z)^{2 n}} f^{k}(z)\right|_{z=0} & =\sum_{\substack{j_{i}, \ldots, j_{k} \geqq 2 \\
\sum_{i=1}^{k} j_{l}=2 n}} \frac{(2 n) !}{j_{1} ! \ldots j_{k} !} \prod_{i=1}^{k} 2^{p j_{i}-1} N^{-1 / 2 j_{i}+1} a_{j_{i}} \\
& =2^{p 2 n-k} N^{-n+k} \sum_{\substack{j_{i}, \ldots, j_{k} \geqq 2 \\
\sum_{i=1}^{k} j_{i}=2 m}} \frac{(2 n) !}{j_{1} ! \ldots j_{k} !} \prod_{i=1}^{k} a_{j_{i}} \\
& \equiv 2^{p 2 n-k} N^{-n+k} b_{n, k} .
\end{aligned}
$$

As follows from the bound on $a_{j}$ the coefficients $b_{n, k}$ are bounded by $2^{4 n} e^{-k\left(2^{-p+1}\right)}$. Summing over $k \leqq n$ we obtain

$$
\begin{aligned}
\left.\frac{d^{2 n}}{(d z)^{2 n}} e^{f(z)}\right|_{z=0} & =\sum_{k=1}^{n} \frac{1}{k !} 2^{p 2 n-k} N^{-n+k} b_{n, k} \\
& =2^{2 p n} \sum_{k=0}^{n-1}\left(\frac{1}{(n-k) !} 2^{-n+k} b_{n, n-k}\right) N^{-k} \\
& =2^{(2 p-1) n} b_{n, n}\left\{1+\sum_{k=1}^{n-1} 2^{k} \frac{b_{n, n-k}}{b_{n, n}} N^{-k}\right\}
\end{aligned}
$$

This together with (A.20) and the estimates on the coefficients $b_{n, k}$ yields Lemma A.3 c).

Acknowledgements. The third author would like to thank Professors J.T. Lewis, R.F. Werner, and N.G. Duffield for interesting discussions and hospitatlity at the IAS-Dublin. We are also grateful to Professor L. Pastur for stimulating remarks and information about his interesting work [12], 
prior to publication. We also thank Professor van Hemmen for his interesting comments and for sending us his papers on related subjects. The hospitality and financial support of the Mathematics Departments of Ruhr-University, Bochum and Rome University, as well as of the SFB 237 Essen-Bochum-Düsseldorf are also gratefully acknowledged.

\section{References}

1. Amit, D., Gutfreund, H., Sompolinsky H.: Spin glass models of neural networks. Phys. Rev. A 32, 1007-1018 (1967)

2. Brascamp, H., Lieb, E.H.: On extension of the Brun-Minkowski and Prekopa-Leindler theorem, including inequalities for log-concave functions and with applications to the diffusion equations. J. Funct. Anal. 22, 266-389 (1976)

3. Combet, E.: Integrales Exponentielles. Lect. Notes in Math vol. 937, Berlin, Heidelberg, New York: Springer 1982

4. Dobrushin, R.L., Zahradnik, M.: Phase Diagram for Continuous Spin Models: An Extension of the Pirogov-Sinai Theory. pp. 1-123 In Mathematical Problems of Statistical Mechanics and Dynamics. Dobrushin, R.L. (ed.) Dordrecht: D. Reidel, 1966

5. Duffield, N.G., Kühn, R.: The thermodynamics of a site-random mean field quantum systems. J. Phys. A22, 4643-4658 (1989)

6. Gawedzki, K., Kupiainen, A., Tirozzi, B.: Borel Summability of the Perturbation Series in a Hierarchical $\lambda(\nabla \phi)^{4}$ Model. J. Stat. Phys. 36, 95-162 (1989)

7. Glimm, J., Jaffe, A.: Quantum physics: A functional integral point of view. Berlin, Heidelberg, New York: Springer 1987, 2nd edition

8. Hopfield, J.J.: Neural networks and physical systems with emergent collective computational abilities. Proc. Nat. Acad. Sci. (USA), 79, 2554 (1982)

9. Kühn, R., van Hemmen, J.L.: Collective phenomena in neutral methods. In: Models of Neural Networks. Domany, E., van Hemmen, J.L., Schulten, K. (eds.) Berlin, Heidelberg, New York: Springer 1991, pp. 1-105

10. Malyshev, W.A., Minlos, R.A.: Gibbsian Random Fields. The Method of Cluster Expansions (in Russian) Moscow: Nauka 1985

11. Mezard, M., Parisi, G., Virasoro, M.: The Spin Glass Theory and Beyond. Singapore: World Scientific 1986

12. Pastur, L., Szczerbina, M.V.: The absence of the selfaverageness of order parameter in the Sherrington-Kirkpatrick model. J. Stat. Phys. 62, 1-19 (1991)

13. Procesi, C., Tirozzi, B.: Metastable states in the Hopfield model. Int. J. Mod. Phys. B4, $143-150$ (1990)

14. Sokal, A.D.: An improvement of Watson's theorem on Borel summability. J. Math. Phys. 21, 261-263 (1980)

15. Raggio, G.A., Werner, R.F.: The Gibbs Variational Principle for Inhomogenous Mean-Field Systems. IAS Dublin, Preprint 1990; Quantum statistical mechanics of general mean field systems. Helv. Phys. Acta. 62, 980-1003 (1989)

16. Van Hemmen, J.L.: Spin-glass methods of neural network. Phys. Rev. A 34, 3435-3445 (1986)

17. Koch, H., Piasko, J.: Some rigorous results on the Hopfield neural network model. J. Stat. Phys. 55, 903-928 (1989)

18. Figotin, A., Pastur, L.: On the theory of disordered spin systems. Theor. Math. Phys. 35, 193-204 (1978)

Communicated by H. Araki 
